\title{
CONDUCTA ALIMENTARIA Y CONSUMO DE ALCOHOL DURANTE EL DISTANCIAMIENTO SOCIAL POR COVID-19 EN MÉXICO: UN ESTUDIO EXPLORATORIO
}

\section{Eating and drinking behavior during social distancing due to COVID-19 in Mexico: an exploratory study}

\section{Lindsay Carrillo Valdez}

Departamento de Investigaciones Educativas. Centro de Investigación y de Estudios Avanzados del Instituto Politécnico Nacional. México.

ORCID: 00 00-0003-4355-0400

Correo-e: psiclcarrillovz@gmail.com

Juan Manuel Reyes Muñiz

Facultad de Estudios Superiores Iztacala. Universidad

Nacional Autónoma de México.

ORCID: 0000-0002-1240-974

Correo-e: juanmanuelreyesm83@gmail.com

\author{
María Luisa Escamilla Gutiérrez \\ Instituto de Ciencias de la Salud. Universidad Autó- \\ noma del Estado de Hidalgo. \\ ORCID: 00 00-0002-6318-4519 \\ Correo-e: marilu.escamilla16@g mail.com \\ Viviana González Baena \\ Centro de Psicoterapia Cognitiva. Nicté Capital \\ Humano. ORCID: 00 00-0002-7021-8983 \\ Correo-e: vigoba828@hotmail.com
}

Recibido: 2/04/2021 • Aprobado: 25/04/2021

Cómo citar: Carrillo Valdez, L., Reyes Muñiz, J. M., Escamilla Gutiérrez, M. L., \& González Baena, V. (2021). Conducta alimentaria y consumo de alcohol durante el distanciamiento social por COVID-19 en México: un estudio exploratorio. Ciencia y Sociedad, 46(2), 7-30. DOI: https://doi.org/10.22206/cys.2021.v46i2.pp7-30

\section{Resumen}

El distanciamiento social por COVID-19 puede ser una experiencia asociada a emociones desagradables y estrés, capaz de exacerbar la ingesta emocional de comida y bebidas alcohólicas como forma de afrontamiento. Con el objetivo de explorar la conducta alimentaria y el consumo de alcohol durante el distanciamiento social por COVID-19 e identificar las variables psicosociales asociadas a dichas conductas, se diseñó un estudio transversal exploratorio, descriptivo y correlacional, con aplicación de una encuesta en línea, a una muestra no probabilística de 1110 mexicanos. A partir del análisis de regresión lineal múltiple, se encontró que las variables: estrés, conductas negativas, ansiedad, sexo femenino, convivencia con un familiar con diagnóstico de COVID-19 y lugar de residencia en el interior de la República Mexicana son factores que predicen el consumo excesivo de comida durante el distanciamiento físico, mientras que el apoyo social es un factor protector $\left(\mathrm{R}^{2}=.286\right)$. Por otra parte, el modelo para el consumo de alcohol durante la cuarentena incluyó las variables estrés y ansiedad como predictores, así como sexo femenino, edad, ocupación y distracción como factores protectores

\section{Abstract}

Social distancing due to COVID-19 may be an experience associated with unpleasant emotions and stress that can exacerbate emotional eating and excessive alcohol use as a way of coping. In order to explore eating and drinking behavior during physical distancing due to COVID-19 and to identify the psychosocial variables associated with these behaviors, an exploratory, descriptive, and correlational cross-sectional study was designed, with the application of an online survey. The non-probability sample consisted of 1,110 Mexicans. The multiple linear regression analysis shows that the variables: stress, negative behaviors, anxiety, female gender, living with a relative with a diagnosis of COVID-19 and place of residence outside Mexico City and of State of Mexico are factors that predict the excessive food consumption, while social support is a protective factor $\left(\mathrm{R}^{2}=.286\right)$. On the other hand, the model for excessive alcohol use during social distancing included the variables: stress and anxiety as predictors, as well as female gender, age, occupation and distraction as protective factors $\left(\mathrm{R}^{2}=.205\right)$. It was concluded that the identified psychosocial variables might contribute to the 
$\left(\mathrm{R}^{2}=.205\right)$. Se concluyó que las variables psicosociales identificadas posibilitan el diseño de estrategias de intervención orientadas a mitigar y/o potenciar el efecto de dichas variables sobre la conducta alimentaria y el consumo de bebidas alcohólicas en situaciones altamente estresantes como la crisis sanitaria actual.

Palabras clave: pandemia COVID-19; salud mental; ingesta alimentaria emocional; consumo excesivo de alcohol.

\section{Introducción}

La pandemia por COVID-19 ha modificado profundamente muchos aspectos de la vida cotidiana (Capasso et al., 2021; Graupensperger et al., 2021; Ramalho, 2020; Zvolensky et al., 2020). El distanciamiento social, la cuarentena y/o el aislamiento son esenciales para el manejo de la crisis sanitaria (American Psychological Association [APA], 2021; Brooks et al., 2020; Natera, 2020; Villanueva et al., 2020). No obstante, muchas personas experimentan esta situación como desagradable, estresante y potencialmente traumática (Comisión Nacional contra las Adicciones [CONADIC], Secretaría de Salud, 2020; Touyz et al., 2020). Varios autores e instituciones coinciden en que la disminución del contacto físico con otras personas, la pérdida de libertad para realizar actividades en el exterior, la incertidumbre sobre el estado de salud, el cierre de espacios públicos, la interrupción y cambios en la rutina, el aburrimiento por permanecer en casa, el estrés, la incapacidad para la autorregulación y la crisis económica pueden tener efectos colaterales, a corto, mediano y largo plazo, en la salud física y psicosocial, especialmente, en grupos vulnerables (APA, 2021; Armitage \& Nellums, 2020; Brooks et al., 2020, Canadian Centre on Substance Use and Addiction; 2020; CONADIC, 2020; Haddad et al., 2020; Hanada et al., 2020; Rehm et al., 2020).

Capasso et al. (2021) sostienen que el escenario pandémico actual se caracteriza por condiciones perturbadoras y altamente estresantes que podrían exacerbar las alteraciones emocionales y el consumo design of intervention strategies aimed at mitigating and / or enhancing the effect of these variables on eating and drinking behavior in highly stressful situations, such as the current health crisis.

Keywords: COVID-19 pandemic; mental health; emotional eating, excessive alcohol consumption.

de alcohol. Adicionalmente, CONADIC (2020) indica que también es probable que se agudicen las creencias mitificadas respecto al uso de sustancias, disminuya la actividad física y aumente el consumo compulsivo de comida. Lo anterior tienen lugar cuando, a través de la ingesta de comida y de bebidas alcohólicas, las personas buscan disminuir los efectos desagradables de una situación estresante y/o generadora de ansiedad, o bien, cuando intentan sentirse con mejor ánimo (Laitinen et al., 2002; CONADIC, 2020). De acuerdo con el modelo transaccional de estrés de Lazarus y Folkman (1984) y el modelo transaccional de afrontamiento de Moos y Holahan (2003, citado en Moos, 2010), tanto factores personales como situacionales interactúan entre sí ante la presencia de un estresor. La acción recíproca de estos factores permite a la persona responder en términos de aproximación vs. evitación y poner en marcha esfuerzos cognitivos y conductuales que constituyen su modo o estilo de afrontamiento (Higareda et al., 2015; Lazarus \& Folkman, 1984). Laitinen et al. (2002) señalan que, generalmente, las personas que recurren a la comida y al alcohol cuando experimentan estrés presentan un afrontamiento centrado en la emoción. Dicho en términos de Moos (2010), son personas que, por un lado, procuran la descarga emocional, esto es, la atenuación de las emociones generadas por la situación estresante y, por el otro, buscan recompensas alternativas. Esto resulta de especial interés en el contexto de COVID-19 en donde el estrés generado por la crisis ocurre a la par del aumento de la disponibilidad física y la asequibilidad de alimentos y bebidas (Rehm et al., 2020). 


\section{Conducta alimentaria y consumo de alcohol durante el distanciamiento social por COVID-19 en México: un estudio exploratorio}

La cuantificación de las alteraciones emocionales y los comportamientos de riesgo asociados a estas es importante para orientar y determinar la eficacia de las políticas de salud emprendidas por las autoridades de cada nación — por ejemplo, las restricciones para la producción, distribución y venta de alcohol-, así como de los programas individuales y grupales de intervención para mitigar el impacto biopsicosocial de la pandemia y promover estilos de vida saludables durante el distanciamiento social y la nueva normalidad (Barbosa et al., 2020a; Rehm et al., 2020). Sin embargo, los datos son limitados y existe falta de consenso sobre la forma en que el distanciamiento físico por COVID-19 afecta la conducta alimentaria y el consumo de alcohol (Ammar et al., 2020; Clay \& Parker, 2020; Mayasari et al., 2020; Villanueva et al., 2021).

La mayor parte de las investigaciones se han llevado a cabo en Europa, Asia y Norteamérica. En cuanto a la conducta alimentaria, actividad física y ganancia de peso, Al-Musharaf (2020) encontró, para mujeres sauditas, correlaciones positivas entre ansiedad y menor actividad física $(\mathrm{r}=.107, \mathrm{p}<.001)$, al igual que para la variable estrés $(\mathrm{r}=.08, \mathrm{p}<.05)$, que también correlacionó con una mayor cantidad de ingesta de refrigerios $(r=0.084, p<0.05)$. Por otro lado, Ammar et al. (2020) realizaron un estudio multi región, con datos provenientes, principalmente, de África, Asia y Europa. Encontraron que la frecuencia de la actividad física disminuyó en un $22.7 \%$ durante el confinamiento $(t=7.75, \mathrm{p}<.001$, $\mathrm{d}=.374)$. Por otro lado, el consumo de comida poco saludable fue mayor $(\mathrm{t}=-3.46, \mathrm{p}<.001, \mathrm{~d}=.14)$, al igual que la ingesta fuera de control $(t=-9.44$, $\mathrm{p}<.001, \mathrm{~d}=0.22)$. Por su parte, Di Renzo et al. (2020) hallaron que el $48.6 \%$ de jóvenes italianos encuestados percibieron aumento en su peso. No obstante, un $38.3 \%$ mencionó haber incrementado su actividad física. Asimismo, el $34.4 \%$ reconoció alteraciones en el apetito predichas por el sexo (OR $=1.521, \mathrm{p}<0,001 ; \mathrm{OR}=1.738, \mathrm{p}<0,001)$. Cabe mencionar que, en Espańa, el $49.8 \%$ de los adultos encuestados también mencionó haber ganado peso durante el confinamiento (López et al., 2020). Mientras tanto, Haddad et al. (2020) encontraron que, en una muestra libanesa, la preocupación por la alimentación se asoció con el género femenino $(\beta=.52)$, mayor ansiedad $(\beta=.04)$, IMC alto $(\beta=.06)$, sensación constante de inseguridad $(\beta=.41)$ y actividad física $(\beta=.43)$. Finalmente, sobre el trastorno por atracón durante el confinamiento, Hanada et al. (2020) hallaron, en una muestra brasileña, que el $22.8 \%$ de los encuestados obtuvo un probable diagnóstico en su forma moderada a grave, el $64.7 \%$ mostró un nivel moderado y el $35.3 \%$ grave.

Ahora bien, el consumo de alcohol en Estados Unidos de América ha sido ampliamente documentado. Los investigadores encontraron un aumento en el consumo de bebidas alcohólicas por día $(+29 \%, \mathrm{p}<.001)$ y consumo excesivo de alcohol $(+21 \%, \mathrm{p}<.001)$ durante el primer mes de confinamiento. En general, el $31 \%$ de los participantes refirió beber alcohol con más frecuencia, mientras que el $21 \%$ aseguró hacerlo con menor asiduidad. Además, hallaron que las mujeres presentaron mayor consumo excesivo que los hombres $(\mathrm{p}=.026)$, aunque estos últimos aumentaron las bebidas por día un poco más que las mujeres (.21 vs. a .19, $\mathrm{p}=$.77) (APA, 2021; Barbosa et al., 2020a, 2020b). Asimismo, Capasso et al. (2021) hallaron asociación entre los síntomas de ansiedad referidos por los participantes y el consumo frecuente de alcohol $(\mathrm{OR}=1.41 ; 95 \% \mathrm{IC}=1.20-1.66 ; \mathrm{p}<.05)$. Adicionalmente, sus hallazgos sugieren que las personas entre 18 y 39 años de edad tienen mayor probabilidad de reportar más consumo de alcohol $(\mathrm{OR}=$ $.44 ; 95 \% \mathrm{IC}=.40, .48 ; \mathrm{p}<.05)$ que las de edad avanzada $(\mathrm{OR}=.73 ; 95 \% \mathrm{IC}=0,63,0,83 ; \mathrm{p}<.05)$. No obstante, Graupensperger et al. (2021) encontraron que los estudiantes universitarios estadounidenses que viven en casa con sus padres durante la pandemia disminuyeron su consumo de alcohol y que la edad y el sexo no se relacionaron con este. 
En la misma línea, el Canadian Centre on Substance Use and Addiction (2020) reportó que el $47 \%$ de un grupo de canadienses encuestados refirió mantener el mismo consumo de alcohol durante la pandemia, pero el $20 \%$ lo aumentó, mientras que solo el $10 \%$ aseguró una disminución. Al igual que los estadounidenses, los residentes de Canadá de entre 18 y 34 años mostraron mayor probabilidad de informar un consumo frecuente (29\%) en comparación con los adultos mayores $(13 \%)$. Además, de acuerdo con la cantidad de alcohol ingerido, se encontró que las mujeres que consumen un trago o dos superaron a los hombres que ingieren la misma cantidad (37\% de los hombres vs. $49 \%$ de las mujeres y $31 \%$ de los hombres vs. $32 \%$ de las mujeres, respectivamente) (Rodríguez et al., 2020).

Para encuestados de África, Europa y Asia, se encontró una disminución significativa en el consumo de alcohol durante el confinamiento $(\mathrm{t}=-12.16$, $\mathrm{p}<.001, \mathrm{~d}=.58$ ) (Ammar et al., 2020). De la misma forma, en España, el consumo de riesgo mostró un descenso importante $(15,1 \%)$, tanto en hombres $\left(\chi^{2}=224.829 ; \mathrm{p}<.001\right)$ como en mujeres $\left(\chi^{2}=214.324 ; \mathrm{p}<.001\right)$, pero los hombres reportaron mayor consumo que las mujeres $\left(\mathrm{t}_{(2343)}=-5.664\right.$; $\mathrm{p}<.001 ; \mathrm{d}=.231)$. A diferencia de Estados Unidos y Canadá, el rango de edad de 18 a 24 ańos fue el que mostró menor prevalencia en consumo, de hecho, el consumo de riesgo durante el confinamiento aumentó conforme incrementó la edad de los participantes $\left(\mathrm{F}_{(5,2338)}=43.849 ; \mathrm{p}=.001\right)$. Además, de acuerdo con la ocupación, el grupo conformado por estudiantes fue el que presentó una menor prevalencia en comparación con el resto $\left(\chi^{2}=8.191 ; \mathrm{p}=.004\right)$ (Villanueva et al., 2021) tal como sucedió en el estudio de Graupensperger et al. (2021).

En México no se encontraron publicaciones que evidencien el estudio científico de la conducta alimentaria durante las fases iniciales de la pandemia por COVID-19. Para el consumo de alcohol, Natera (2020), sostiene que los hombres aumentaron la ingesta de bebidas alcohólicas por ansiedad en un $47 \%$, mientras que las mujeres lo hicieron en un $56 \%$. Además, los nuevos consumidores alcanzaron el $5.5 \%$ durante la pandemia. En cuanto a la frecuencia de consumo, tanto los hombres (18.9\% vs. $7.8 \%)$ como las mujeres ( $15.3 \%$ vs. $3.9 \%)$ aumentaron el número de días de uso de alcohol con respecto al reportado antes de la pandemia, mientras que la frecuencia referida al consumo una vez a la semana disminuyó para ambos sexos (hombres = $47.7 \%$ vs. $64 \%$ y mujeres $=51.9 \%$ vs. $59.8 \%$ ).

Aunque ha circulado un buen número de encuestas en línea orientadas a la evaluación de variables psicosociales, conducta alimentaria, consumo de alcohol y otras sustancias durante la pandemia en México, se desconocen las metodologías empleadas, así como los resultados obtenidos. Por tanto, ante la limitada evidencia empírica y bajo el supuesto de que el distanciamiento social exacerba la ingesta emocional de comida y bebidas alcohólicas como forma de afrontamiento ante el estrés, se planteó como objetivo de investigación explorar la conducta alimentaria y el consumo de alcohol durante el distanciamiento social por COVID-19 e identificar las variables psicosociales asociadas a dichas conductas. Para ello, y dadas las condiciones sanitarias, se optó por un estudio exploratorio con estrategia metodológica cuantitativa, recolección remota de datos y por autoinforme, así como análisis estadístico descriptivo e inferencial.

\section{Método}

Diseño

Estudio transversal, exploratorio, descriptivo y correlacional. 


\section{Conducta alimentaria y consumo de alcohol durante el distanciamiento social por COVID-19 en México: un estudio exploratorio}

\section{Participantes}

Debido a las medidas nacionales de distanciamiento social para el control del brote epidémico, se eligió un muestreo no probabilístico tipo bola de nieve. Se diseñó y aplicó una encuesta en línea a través de Google Forms ${ }^{\circledast}$. El índice de respuesta obtenido fue del $96.5 \%$, ya que 51 personas no proporcionaron su consentimiento para el uso de datos. Además, se excluyó la información de 290 participantes menores de edad y 25 más que reportaron residencia fuera de México. Adicionalmente, 48 datos no fueron considerados debido a la detección de repeticiones y omisiones en la información sociodemográfica. Por tanto, la muestra final estuvo constituida por 1110 mexicanos (62.8 \% mujeres y $37.2 \%$ hombres). El promedio de edad fue de 26.83 ańos ( $\mathrm{DE}=11.346$ ). La mayoría refirió residencia en el Estado de México (64.2\%), estado civil soltero (67.5\%), nivel de estudios medio superior (52.5\%), ocupación estudiante ( $44.8 \%$ ), tener con algún tipo de creencias religiosas $(69.2 \%)$ y contar con seguridad social (69.2\%).

Sobre su estado de salud física, gran parte de la muestra lo evaluó como bueno (36.4\%), sin enfermedades crónicas $(81.6 \%$ ) y sin síntomas $(90.7 \%$ ) o diagnóstico de COVID-19 (97 \%) al momento del estudio. En cuanto a sus condiciones de vida, la mayor parte de la muestra aseguró ingresos económicos familiares mensuales entre $\$ 5$ mil y $\$ 10 \mathrm{mil}$ pesos mexicanos $(36.3 \%)$, habitar en hogares conformados por 3 a 4 personas (47.7\%), convivir con niños menores de 12 ańos (55.3\%), sin presencia de mujeres embarazadas (95.9 \%) ni adultos mayores (66.3\%) en el hogar. Además, aseveraron cohabitar con familiares diagnosticados con alguna enfermedad crónico-degenerativa $(56.8 \%)$ sin síntomas (62.3 \%) o diagnóstico de COVID-19 (64.8 \%). La tabla 1 resume las principales características sociodemográficas de los participantes.
Tabla 1. Caracteristicas sociodemográficas de la muestra $(n=1110)$

\begin{tabular}{|c|c|c|c|}
\hline Variable & Categoría & $\mathrm{F}$ & $\%$ \\
\hline \multirow{2}{*}{ Sexo } & Mujer & 697 & 62.8 \\
\hline & Hombre & 413 & 37.2 \\
\hline \multirow{3}{*}{$\begin{array}{l}\text { Edad } \\
M E=26.83 \\
D E=11.346\end{array}$} & Adultez temprana & 961 & 86.6 \\
\hline & Adultez intermedia & 134 & 12.1 \\
\hline & Adultez tardía & 15 & 1.3 \\
\hline \multirow{6}{*}{ Estado civil } & Soltero & 749 & 67.5 \\
\hline & Casado & 177 & 15.9 \\
\hline & Unión libre & 109 & 9.8 \\
\hline & Separado & 39 & 3.5 \\
\hline & Divorciado & 28 & 2.5 \\
\hline & Viudo & 8 & 0.8 \\
\hline \multirow{3}{*}{$\begin{array}{l}\text { Lugar de } \\
\text { residencia }\end{array}$} & Ciudad de México & 282 & 25.4 \\
\hline & Estado de México & 713 & 64.2 \\
\hline & Interior de la República & 115 & 10.4 \\
\hline \multirow{4}{*}{ Escolaridad } & Básico & 87 & 7.8 \\
\hline & Medio superior & 583 & 52.5 \\
\hline & Superior & 392 & 35.3 \\
\hline & Posgrado & 48 & 4.4 \\
\hline \multirow{5}{*}{ Ocupación } & Actividad remunerada & 422 & 38 \\
\hline & Hogar & 91 & 8.2 \\
\hline & Estudiante & 497 & 44.8 \\
\hline & Jubilado & 13 & 1.2 \\
\hline & Desempleado & 87 & 7.8 \\
\hline \multirow{4}{*}{$\begin{array}{l}\text { Número de } \\
\text { personas que } \\
\text { viven en el } \\
\text { hogar }\end{array}$} & 1 a 2 & 118 & 10.6 \\
\hline & 3 a 4 & 530 & 47.7 \\
\hline & 5 a 7 & 314 & 28.3 \\
\hline & 7 o más & 148 & 13.3 \\
\hline \multirow{4}{*}{$\begin{array}{l}\text { Ingreso } \\
\text { económico } \\
\text { familiar por } \\
\text { mes (en pesos } \\
\text { mexicanos) }\end{array}$} & Menos de $\$ 5$ mil & 311 & 28 \\
\hline & $\begin{array}{l}\text { Más de } \$ 5 \text { mil, menos de } \\
\$ 10 \text { mil }\end{array}$ & 403 & 36.3 \\
\hline & $\begin{array}{l}\text { Más de } \$ 10 \text { mil, menos } \\
\text { de } \$ 15 \text { mil }\end{array}$ & 195 & 17.6 \\
\hline & Más de $\$ 15$ mil & 201 & 18.1 \\
\hline
\end{tabular}

Nota. COVID-19 = Coronavirus disease. La variable edad fue agrupada en tres categorías de acuerdo con las etapas de la adultez propuestas por Feldman (2007). 


\section{Instrumentos}

Ante las restricciones para la observación directa de conductas, se digitalizaron seis instrumentos de autoinforme distribuidos en tres secciones. La primera se denominó datos sociodemográficos y constó de un cuestionario de dieciocho preguntas ad hoc.

El segundo apartado, llamado variables psicológicas, midió estrés, ansiedad fisiológica ante el coronavirus y estilos de afrontamiento. Para la variable estrés se recurrió a la Perceived Stress Scale de 10 ítems (PSS-10), versión adaptada y validada $(\alpha=.83)$ por Velasco (2017) para población mexicana. La escala parte de la concepción del estrés psicológico como resultado de la interacción persona-ambiente en la que las exigencias de este último son percibidas como excedentes de los recursos disponibles (Lazarus y Folkman, 1984). Así, evalúa dos factores: 1) distrés (estrés negativo percibido) y 2) capacidad de afrontamiento (gestión del estrés). Ofrece cinco opciones de respuesta $(0=$ nunca a $4=$ muy frecuentemente), de ahí que el puntaje mínimo a obtener es 0 y el máximo 40. Los puntajes altos se relacionan con mayor estrés percibido. Además, las puntuaciones se pueden clasificar en niveles de estrés bajo ( 0 a 13 puntos), moderado (14 a 26 puntos) y alto (27 a 40 puntos). Adicionalmente, se incluyó un reactivo en el que se solicitó la elección del factor considerado como mayor generador de estrés durante la Fase 3 de la pandemia de COVID-19 en México.

La variable ansiedad fisiológica ante el coronavirus se midió a través de la Coronavirus Anxiety Scale (CAS-4SP), versión en español, adaptada y validada $(\alpha=.792 ; \omega=.868$; sensibilidad $=91 \%$, especificidad $=96.9 \%)$ por Carrillo-Valdez (2020). La escala considera cuatro síntomas somáticos desa- gradables que se experimentan ante situaciones de peligro como las relacionadas con el SARS-CoV-2. Consta de cuatro ítems y presenta cuatro opciones de respuesta $(0=$ de ningún modo; $1=$ rara vez, menos de un día o dos; 3 = varios días; 3 = más de 7 días y 4 = casi todos los días durante las últimas 2 semanas). El puntaje mínimo es 0 y el máximo es de 16 puntos. Para las mujeres sin síntomas de COVID-19 el punto de corte es $\geq 4$, mientras que para los hombres un puntaje $\geq 3$ se considera clínicamente relevante. Ante presencia de síntomas de COVID-19, el punto de corte para mujeres es de $\geq 5$ y para hombres es $\geq 6$. Asimismo, la escala permite la clasificación de los niveles de ansiedad en mínima, leve, moderada, alta y muy alta.

Los estilos de afrontamiento, concebidos como los modos en que la persona pone en marcha esfuerzos cognitivos y conductuales ante situaciones estresantes (Lazarus \& Folkman, 1984), se evaluaron mediante la Scale of Moos Type-B Coping Styles (B-Moos), versión adaptada y validada para población mexicana por Higareda et al. (2015). Esta versión está conformada por 35 ítems y ofrece seis opciones de respuesta. En las primeras dos dimensiones las alternativas de respuesta van de $0=$ nunca a $6=$ siempre a casi siempre (más del $80 \%$ del tiempo), mientras que la tercera ofrece como opciones $0=$ ninguno a 6 = más de cuatro. La distribución de los reactivos en las tres dimensiones es la siguiente: a) estilos de afrontamiento personal $(\alpha=.82)$ que incluye las subescalas estilo de afrontamiento cognitivo $(0 \mathrm{a}$ 35 puntos); baja tolerancia a la frustración y retirada social (0 a 20 puntos); afrontamiento externo (0 a 24 puntos); preparación para la acción (0 a 20 puntos); evitación del malestar (0 a 20 puntos); distracción (0 a 20 puntos); b) conductas negativas de afrontamiento con puntaje mínimo de 0 y máximo de $20(\alpha=.71)$ y c) red de apoyo $(\alpha=.81)$ con 20 puntos como máximo posible. Los puntajes 


\section{Conducta alimentaria y consumo de alcohol durante el distanciamiento social por COVID-19 en México: un estudio exploratorio}

altos en cada subescala indican mayor probabilidad de un foco (orientación) y de ejecutar un método (esfuerzos determinados) ante situaciones estresantes (Moos, 2010).

La tercera sección fue nombrada excesos conductuales y evaluó los cambios percibidos en la conducta alimentaria, actividad física y peso, la ingesta excesiva de comida y el consumo de alcohol. Para la primera dimensión se presentaron cinco reactivos referidos a la valoración de los hábitos alimentarios durante el distanciamiento social, así como el mantenimiento, aumento o disminución percibida en el consumo de alimentos, comida poco saludable, actividad física y peso.

La ingesta excesiva de comida, esto es, episodios de consumo de grandes cantidades de alimentos, se evaluó a través del Binge Eating Disorder Screener-7 (BEDS-7), versión adaptada y traducida para México por Grau (2014). Presenta siete reactivos con opción de respuesta dicotómica (Sí y No), donde tres o más respuestas afirmativas se relacionan con la probabilidad de trastorno por atracón. El instrumento no cuenta con datos de validación; sin embargo, para el presente estudio la escala mostró confiabilidad aceptable $(\alpha=.784)$.

Finalmente, el consumo de alcohol, es decir, ingerir bebidas con graduación etílica, se midió mediante un reactivo referido a la percepción de cambios en el consumo de bebidas alcohólicas durante el distanciamiento social, un reactivo para la elección de tipos de bebidas alcohólicas ingeridas, así como la administración del Alcohol Use Disorders Identifcation Test (AUDIT), un instrumento desarrollado por Babor et al. (2001) cuyo empleo en México es ampliamente difundido. De acuerdo con Villamil et al. (2009) las adaptaciones mexicanas muestran sensibilidad (80 \%) y especificidad (89\%) altas, así como consistencia interna adecuada $(\alpha=.87)$. Consta de 10 reactivos cuyas opciones de respuesta fluctúan entre 0 y 4 puntos. Permite la clasificación del consumo de alcohol a través de tres categorías: a) 0 a 3 puntos = consumo responsable; b) 4 a 7 puntos $=$ consumo de riesgo o perjudicial y c) $8 \mathrm{o}$ más puntos $=$ abuso o dependencia.

\section{Procedimiento}

El estudio tuvo lugar entre el 18 y 31 de mayo de 2020, es decir, durante la Fase 3 de la pandemia en México. En ese momento, de acuerdo con las autoridades sanitarias y gubernamentales mexicanas, se vislumbraba el retorno a actividades (nueva normalidad) a partir del 1 ero de junio para algunas regiones. Con el fin de respetar las disposiciones de sana distancia, se enviaron invitaciones digitales a participantes potenciales identificados en redes sociales y comunidades virtuales universitarias (Facebook, WhatsApp y Messenger). Las invitaciones contenían el enlace al formulario de Google ${ }^{\circ}$, así como la petición para compartir entre sus contactos.

Aunque el cuestionario empleó el modo de respuesta forzada para evitar la pérdida de datos y garantizar la aceptación del consentimiento informado antes del envío, el participante podía retirarse del estudio en el momento que lo decidiera. Cabe resaltar que, debido a las características de las variables evaluadas, el consentimiento informado fue presentado de forma detallada y se incluyeron datos de contacto (correo electrónico) ante la posibilidad de que algunos reactivos causaran inquietud y/o demanda de información, orientación y/o psicoeducación gratuita. Además, se proporcionaron enlaces a sitios de internet con información confiable y útil para disminuir algunos efectos psicosociales (ansiedad, estrés, ingesta excesiva de comida y consumo de alcohol) derivados de las medidas de prevención 
y control de la COVID-19. Asimismo, no se solicitó información que pudiera contribuir a la identificación de los participantes, con el propósito de garantizar la confidencialidad de sus datos. Todo lo anterior con el fin de cumplir los criterios éticos internacionales de la Declaración de Helsinki que se aplican a los estudios psicológicos sin intervención. Además, ante la falta de constancia del consentimiento de padres y/o tutores, la información de participantes menores de edad fue excluida.

\section{Análisis estadístico}

Descriptivo e inferencial con el programa SPSS, versión 25. Cabe señalar que los factores sociodemográficos fueron utilizados tanto como variables independientes como dependientes.

\section{Resultados}

Respecto de las percepciones sobre la conducta alimentaria, actividad física y peso durante el distanciamiento social por COVID-19 la muestra refirió pocos cambios. De este modo, la mayoría de los participantes calificó sus hábitos de alimentación como buenos (41.9\%), el $38.3 \%$ los consideró regulares, el $10.1 \%$ excelentes, mientras que el $9.7 \%$ los etiquetó como malos. Asimismo, el $45.8 \%$ consideró que su consumo de comida se mantuvo igual durante la cuarentena, mientras que el $39.4 \%$ percibió un aumento y el $14.9 \%$ señaló una disminución. Aunado a lo anterior, el $35.9 \%$ refirió que su consumo de comida poco saludable durante este periodo fue el mismo; en cambio, el $32.2 \%$ reportó aumento y el $14.9 \%$ disminución. De manera similar, el $40.7 \%$ consideró que su actividad física durante el distanciamiento social disminuyó, mientras que el $30.1 \%$ refirió que se mantuvo igual y el $29.2 \%$ aseguró que incrementó.
Finalmente, respecto del peso corporal, la mayoría (42.4\%) reportó mantenimiento, el $41.5 \%$ notó un aumento y el $16 \%$ percibió disminución.

Ahora bien, en la muestra estudiada, el puntaje promedio obtenido en el BEDS-7 para la ingesta excesiva de comida fue de $2.05(\mathrm{DE}=2.371)$ de 7 puntos máximos. Al tratarse de un puntaje bajo, la probabilidad de desarrollar trastorno por atracón durante el distanciamiento social es igualmente baja entre los participantes. De hecho, la mayor parte de la muestra (63.7\%), al obtener menos de tres puntos, se considera sin riesgo. En cambio, el $36.3 \%$ presentó conductas alimentarias asociadas con un probable trastorno por atracón, ya que obtuvo 4 o más puntos en el instrumento.

Aunado a lo anterior, se observó que los participantes de sexo femenino ( $\mathrm{ME}=2.52 ; \mathrm{DE}=2.51)$, en adultez temprana $(\mathrm{ME}=2.16 ; \mathrm{DE}=2.4)$, solteros $(\mathrm{ME}=2.21 ; \mathrm{DE}=2.41)$, residentes del interior de la República Mexicana $(\mathrm{ME}=2.54 ; \mathrm{DE}=2.53)$, con escolaridad media superior $(\mathrm{ME}=2.18 ; \mathrm{DE}=2.37)$, estudiantes $(\mathrm{ME}=2.45 ; \mathrm{DE}=2.45)$, con ingresos familiares mensuales menores a $\$ 5$ mil pesos mexicanos $(\mathrm{ME}=2.28 ; \mathrm{DE}=2.44)$, que percibieron un mal estado de salud $(\mathrm{ME}=2.38 ; \mathrm{DE}=2.66)$, que contaban con diagnóstico previo de enfermedades crónicas $(\mathrm{ME}=2.67 ; \mathrm{DE}=2.65)$, síntomas $(\mathrm{ME}$ $=2.81 ; \mathrm{DE}=2.59)$ o diagnóstico de COVID-19 $(\mathrm{ME}=2.39 ; \mathrm{DE}=2.61)$ y además convivían con familiares diagnosticados con enfermedades crónicas $(\mathrm{ME}=2.26 ; \mathrm{DE}=2.47)$, síntomas $(\mathrm{ME}=2.27 ; \mathrm{DE}$ = 2.54) o diagnóstico de COVID-19 $(\mathrm{ME}=2.37$; $\mathrm{DE}=2.57)$ obtuvieron puntuaciones más altas que el resto de los grupos (ver apéndice 1). Como muestra la tabla 2, las diferencias y asociaciones encontradas fueron significativas $(\mathrm{p}<.05)$. 


\section{Conducta alimentaria y consumo de alcohol durante el distanciamiento social por COVID-19 en México: un estudio exploratorio}

Tabla 2. Diferencias y asociaciones de las conductas ingesta excesiva de comida y consumo de alcohol durante el distanciamiento social por COVID-19 de acuerdo con variables sociodemográficas

\begin{tabular}{lcccc}
\hline \multirow{2}{*}{ Variable } & \multicolumn{2}{c}{ COM } & \multicolumn{2}{c}{ ALC } \\
& $\mathrm{t}$ o F & $\chi^{2}$ & $\mathrm{t}$ o F & $\chi^{2}$ \\
Sexo & $7.35^{* * *}$ & $53.39^{* * *}$ & $-4.401^{* * *}$ & $63.86^{* * *}$ \\
Edad & $7.92^{* * *}$ & $31.029^{* * *}$ & $14.823^{* * *}$ & $41.62^{* * *}$ \\
Estado civil & $3.45^{* *}$ & 39.27 & $3.944^{* *}$ & 121.74 \\
Lugar de residencia & $3.385^{*}$ & $26.83^{*}$ & 2.437 & 42.41 \\
Escolaridad & $2.666^{*}$ & $46.97^{* * *}$ & $3.722^{* *}$ & 93.03 \\
Ocupación & $6.641^{* * *}$ & $60.44^{* * *}$ & $2.754^{*}$ & $145.38^{* *}$ \\
Creencias religiosas & -0.562 & 3.56 & -1.708 & $52.38^{* *}$ \\
Seguridad social & -1.453 & 10.35 & -0.299 & 23.35 \\
Estado de salud percibido & $15.111^{* * *}$ & $88.35^{* * *}$ & 0.663 & 106.6 \\
Diagnóstico previo de enfermedades crónicas & $3.771^{* * *}$ & $28.15^{* * *}$ & -1.123 & 20.79 \\
Síntomas de COVID-19 & $3.120^{* *}$ & 13.73 & 0.966 & 36.57 \\
Adultos mayores en el hogar & -1.361 & 4.35 & $-2.083^{*}$ & 39.27 \\
Enfermedades crónicas diagnosticadas en familiares que comparten & $3.439^{* * *}$ & 16.33 & -0.264 & 27.63 \\
el hogar & & & & \\
Síntomas de COVID-19 en familiares que comparten el hogar & $2.298^{*}$ & $16.53^{*}$ & 0.062 & 34.33 \\
Diagnóstico de COVID-19 en familiares que comparten el hogar & $3.141^{* *}$ & $21.39^{* *}$ & -1.55 & 36.21 \\
Ingreso económico familiar por mes (en pesos mexicanos) & $3.046^{*}$ & 27.52 & 0.227 & $109.59^{* *}$ \\
\hline
\end{tabular}

Nota. COVID-19 = Coronavirus disease. $\mathrm{COM}=$ Ingesta excesiva de comida; $\mathrm{ALC}=$ Consumo de alcohol. ${ }^{*} \mathrm{p}<.05$

${ }^{* *} \mathrm{p}<.01$

${ }^{* * *} \mathrm{p}<.001$

En cuanto al consumo de alcohol durante el distanciamiento social, mientras el $25.8 \%$ aseguró no consumir alcohol, el 39.2\% refirió ingerir distintas combinaciones de bebidas alcohólicas, así como cerveza (17.6\%), destilados (11.4\%), vino (4.9\%) y pulque $(1.7 \%)$. El $45.9 \%$ de los encuestados señaló una disminución en el uso de alcohol, el $44.9 \%$ reportó que permaneció igual y solo el 9.3 $\%$ mencionó que percibió un aumento. Respecto del consumo excesivo de bebidas con graduación etílica, la muestra registró un promedio de 4.16 puntos $(\mathrm{DE}=4.755)$ en el AUDIT, lo que corresponde, en general, a un consumo de riesgo. No obstante, la mayoría (58.6 \%) se ubicó en el rango de consumo responsable, mientras que el $22.8 \%$ presentó consumo de riesgo y el $18.6 \%$ consumo perjudicial o dependencia. Los participantes de sexo masculino $(\mathrm{ME}=5.03 ; \mathrm{DE}=5.49)$, en adultez temprana $(\mathrm{ME}=4.46 ; \mathrm{DE}=4.91)$, solteros $(\mathrm{ME}$ $=4.52 ; \mathrm{DE}=4.86)$, con escolaridad media superior $(\mathrm{ME}=4.58 ; \mathrm{DE}=5.07)$, desempleados $(\mathrm{ME}=4.4$; $\mathrm{DE}=5.11)$, sin creencias religiosas $(\mathrm{ME}=4.53$; $\mathrm{DE}=4.64)$, con ingresos mensuales entre $\$ 10 \mathrm{mil}$ y $\$ 15$ mil pesos mexicanos $(\mathrm{ME}=4.38 ; \mathrm{DE}=5.48)$ y que convivían con adultos mayores en el hogar $(\mathrm{ME}=4.59 ; \mathrm{DE}=5.11)$ obtuvieron puntajes significativamente $(\mathrm{p}<.05)$ más altos que el resto de los grupos (ver apéndice 1). Nuevamente, en la tabla 2 se observan las diferencias y asociaciones de la conducta consumo excesivo de alcohol durante el distanciamiento social por COVID-19 de acuerdo con factores sociodemográficos. 
En las variables psicológicas los participantes obtuvieron puntuaciones bajas a medias. Particularmente, en ansiedad fisiológica ante el coronavirus el puntaje promedio obtenido en la CAS-4SP fue bajo $(\mathrm{ME}=2.04 ; \mathrm{DE}=2.54$ puntos $)$. De hecho, el $56.7 \%$ reportó niveles mínimos de ansiedad. Sin embargo, el $14.1 \%$ se ubicó en el nivel más alto, seguido del nivel moderado (11.7\%), leve (9.1) y alto (8.5\%). Los niveles de ansiedad fisiológica obtenidos por la muestra evidenciaron diferencias significativas $(\mathrm{p}<.05)$ por sexo, creencias religiosas, estado de salud percibido, presencia de síntomas de COVID-19, número de personas que viven en el hogar, presencia de mujeres embarazadas y adultos mayores en el hogar, así como familiares con síntomas y/o diagnóstico de COVID-19 (ver apéndice 1).

En lo que concierne al estrés psicológico, los puntajes generales obtenidos en la PSS-10 fueron bajos a medios $(\mathrm{ME}=17.86 ; \mathrm{DE}=7.02$ de 40 puntos máximos). El $57.8 \%$ de la muestra obtuvo un nivel moderado, el $33.3 \%$ bajo y el $8.8 \%$ registró un grado de estrés alto. En la dimensión distrés de la PSS-10, los participantes obtuvieron un promedio de $11.62(\mathrm{DE}=5.16)$ de 24 puntos posibles, es decir, el estrés negativo fue percibido entre bajo y moderado. En cambio, en la dimensión afrontamiento la media fue de $9.76(\mathrm{DE}=2.84)$ de 16 puntos posibles, lo que parece indicar que los encuestados percibieron capacidades suficientes para el manejo del estrés psicológico durante la Fase 3 de la pandemia. Las variables sexo, edad, estado civil, lugar de residencia, escolaridad, ocupación, creencias religiosas, seguridad social, estado de salud percibido, síntomas de COVID-19, número de personas que viven en el hogar, ingresos familiares mensuales, así como enfermedades crónicas y síntomas de COVID-19 en familiares que viven en el hogar mostraron diferencias significativas $(\mathrm{p}<.05)$ en los puntajes de estrés psicológico comparadas con otros grupos (ver apéndice 1)

Adicionalmente, en cuanto a los principales estresores identificados por la muestra para el distanciamiento social por COVID-19, la mayoría (22.4\%) refirió a la posibilidad de contagio y muerte de un familiar como el motivo principal de estrés; seguido de la extensión de la cuarentena (15.2\%); la crisis económica (13.4\%); las clases en línea (12.4\%); el contagio y/o muerte personal $(6.8 \%)$, así como no tener empleo $(6.6 \%)$. El resto $(22.9 \%)$ se distribuyó en otras opciones de respuesta que mostraron baja frecuencia.

En estilos de afrontamiento, los participantes obtuvieron puntuaciones medias. Por ejemplo, para el tipo cognitivo el promedio fue de 24.57 puntos $(\mathrm{DE}=6.885)$ de 35 posibles; para baja tolerancia a la frustración y retirada la media obtenida por los encuestados fue de 7.09 puntos $(\mathrm{DE}=4.234)$ de 20 máximos; en afrontamiento externo puntuaron un promedio de $12.74(\mathrm{DE}=5.208)$ de 25 posibles; para el tipo prepararse para la acción el promedio fue de 12.81 puntos $(\mathrm{DE}=3.846$ ) de 20 máximos; en evitación registraron una media de 11.91 puntos $(\mathrm{DE}=3.569)$ de 20 máximos; en distracción el promedio fue de 12.87 puntos $(\mathrm{DE}=3.482)$ de 20 posibles; para afrontamiento conductual (conductas negativas) reportaron una media de 7.85 puntos $(\mathrm{DE}=4.337)$ de 20 máximos; mientras que en red de apoyo la media fue de 8.35 puntos $(\mathrm{DE}=3.86)$ de 15 posibles. Con base en lo anterior, se encontró que los participantes encuestados prefirieron el estilo de afrontamiento cognitivo, distracción y prepararse para la acción ante el distanciamiento social por COVID-19. Tanto el afrontamiento cognitivo como prepararse para la acción corresponden a afrontamiento centrado en el problema, mientras que la distracción es un estilo de afrontamiento orientado a la emoción. Cabe mencionar que las diferencias en los puntajes de estilo de afrontamiento de acuerdo con las variables sociodemográficas evaluadas se observan en el apéndice 1.

Por otro lado, en la tabla 3 se presentan las correlaciones entre variables psicosociales (edad, ansiedad fisiológica, estrés psicológico y afrontamiento) y las conductas ingesta excesiva de comida y consumo de alcohol durante el distanciamiento social por COVID-19. La mayoría de los coeficientes de 


\section{Conducta alimentaria y consumo de alcohol durante el distanciamiento social por COVID-19 en México: un estudio exploratorio}

correlación $(r)$ resultaron significativos y fluctuaron entre débiles y fuertes. Destacan las relaciones positivas entre la ingesta excesiva de comida y estrés $(r=.432, \mathrm{p}<.001)$, ansiedad $(r=.311, \mathrm{p}<.0001)$, baja tolerancia a la frustración y retirada social $(r=$ $.340, \mathrm{p}<.0001)$ y conductas negativas $(r=.351$, $\mathrm{p}<.0001)$. Las relaciones negativas tuvieron lugar, principalmente, con las variables afrontamiento $(r=$ $-.322, \mathrm{p}<.0001)$, estilo de afrontamiento cognitivo $(r=-.236, \mathrm{p}<.0001)$ y apoyo social $(r=-.161$, $\mathrm{p}<.0001)$. Igualmente, se hallaron relaciones positivas entre el consumo excesivo de alcohol y estrés $(r=.129, \mathrm{p}<.0001)$, ansiedad $(r=.225, \mathrm{p}<.0001)$, conductas negativas $(r=.361, \mathrm{p}<.0001)$ e ingesta excesiva de alimentos $(r=.188, \mathrm{p}<.0001)$. En contraste, las principales relaciones negativas se presentaron con afrontamiento $(r=-.125, \mathrm{p}<.0001)$, afrontamiento cognitivo $(r=-.226, \mathrm{p}<.0001) \mathrm{y}$ distracción $(r=-.102, \mathrm{p}<.0001)$.

Tabla 3. Correlaciones entre variables psicosociales, conducta alimentaria y consumo de alcohol durante el distanciamiento social por COVID-19

\begin{tabular}{|c|c|c|c|c|c|c|c|c|c|c|c|c|c|c|c|}
\hline Variable & EDA & ANS & DIST & AFR & EST & $\begin{array}{c}\mathrm{AF} \\
\mathrm{COG}\end{array}$ & $\begin{array}{c}\text { AF } \\
\text { FRUST }\end{array}$ & AF EXT & AF ACC & AF EVI & $\begin{array}{l}\text { AF } \\
\text { DIST }\end{array}$ & $\begin{array}{c}\mathrm{AF} \\
\text { CONEG }\end{array}$ & APO & $\mathrm{COM}$ & ALC \\
\hline EDA & - & & & & & & & & & & & & & & \\
\hline ANS & -0.038 & - & & & & & & & & & & & & & \\
\hline DIST & $-.297^{* * *}$ & $.331^{* * *}$ & - & & & & & & & & & & & & \\
\hline AFR & $.307^{* * *}$ & $-.156^{* * *}$ & $-.459^{* * *}$ & - & & & & & & & & & & & \\
\hline EST & $-.347^{* * *}$ & $.309^{* * *}$ & $.928^{* * *}$ & $-.756^{* * *}$ & - & & & & & & & & & & \\
\hline AF COG & $.250^{* * *}$ & $-.162^{* * *}$ & $-.246^{* * *}$ & $.531^{* * *}$ & $-.404^{* * *}$ & - & & & & & & & & & \\
\hline $\begin{array}{l}\text { AF } \\
\text { FRUST }\end{array}$ & $-.249^{* * *}$ & $.301^{* * *}$ & $.504^{* * *}$ & $-.319^{* * *}$ & $.504^{* * *}$ & $-.257^{* * *}$ & - & & & & & & & & \\
\hline AF EXT & $.257^{* * *}$ & $.112^{* * *}$ & $-.106^{* * *}$ & $.328^{* * *}$ & $-.215^{* * *}$ & $.461^{* * *}$ & $-.114^{* * *}$ & - & & & & & & & \\
\hline AF ACC & $.282^{* * *}$ & 0.01 & $-.183^{* * *}$ & $.440^{* * *}$ & $-.319^{* * *}$ & $.589^{* * *}$ & $-.126^{* * *}$ & $.528^{* * *}$ & - & & & & & & \\
\hline AF EVI & $.106^{* * *}$ & $-.140^{* * *}$ & $-.207^{* * *}$ & $.333^{* * *}$ & $-.292^{* * *}$ & $.407^{* * *}$ & $-.115^{* * *}$ & $.171^{* * *}$ & $.392^{* * *}$ & - & & & & & \\
\hline $\begin{array}{l}\text { AF } \\
\text { DIST }\end{array}$ & $.110^{* * *}$ & -0.058 & $-.130^{* * *}$ & $.306^{* * *}$ & $-.224^{* * *}$ & $.521^{* * *}$ & $-.094^{* *}$ & $.288^{* * *}$ & $.463^{* * *}$ & $.498^{* * *}$ & - & & & & \\
\hline $\begin{array}{l}\text { AF } \\
\text { CONEG }\end{array}$ & $-.254^{* * *}$ & $.267^{* * *}$ & $.309^{* * *}$ & $-.197^{* * *}$ & $.310^{* * *}$ & $-.135^{* * *}$ & $.348^{* * *}$ & 0.024 & $-.078^{* *}$ & -0.034 & 0.033 & - & & & \\
\hline APO & $.177^{* * *}$ & -0.051 & $-.143^{* * *}$ & $.269^{* * *}$ & $-.218^{* * *}$ & $.351^{* * *}$ & $-.217^{* * *}$ & $.343^{* * *}$ & $.265^{* * *}$ & $.176^{* * *}$ & $.209^{* * *}$ & $-.088^{* * *}$ & & & \\
\hline $\mathrm{COM}$ & $-.167^{* * *}$ & $.311^{* * *}$ & $.404^{* * *}$ & $-.322^{* * *}$ & $.432^{* * *}$ & $-.236^{* * *}$ & $.340^{* * *}$ & $-.096^{* * *}$ & $-.128^{* * *}$ & $-.163^{* * *}$ & $-.080^{* *}$ & $.351^{* * *}$ & $-.161^{* * *}$ & - & \\
\hline ALC & $-.194^{* * *}$ & $.225^{* * *}$ & $.105^{* * *}$ & $-.125^{* * *}$ & $.129^{* * *}$ & $-.226^{* * *}$ & $.155^{* * *}$ & $-.074^{* *}$ & $-.142^{* * *}$ & $-.085^{* * *}$ & $-102^{* * *}$ & $.361^{* * *}$ & $-.081^{* *}$ & $.188^{* *}$ & - \\
\hline
\end{tabular}

Nota COVID-19 = Coronavirus disease. EDA = Edad; DDS = Días de distanciamiento social; ANS = Ansiedad fisiológica; DIST = Distrés psicológico; $\mathrm{AFR}=$ Afrontamiento del estrés; $\mathrm{EST}=$ Estrés psicológico; $\mathrm{AF}$ COG = Estilo de afrontamiento cognitivo; AF FRUST = Estilo de afrontamiento baja tolerancia a la frustración y retirada social; AF EXT = Estilo de afrontamiento externo; $\mathrm{AF}$ ACC = Estilo de afrontamiento preparación para la acción; AF EVI = Estilo de Afrontamiento evitación del malestar; AF DIST = Estilo de afrontamiento distracción; AF CONEG = Afrontamiento conductual (conductas negativas); $\mathrm{APO}=$ Red de apoyo social; $\mathrm{COM}=$ Ingesta excesiva de comida ALC = Consumo de alcohol

$$
\begin{aligned}
& { }^{* *} \mathrm{p}<.01 \\
& { }^{* * *} \mathrm{p}<.001
\end{aligned}
$$


Los resultados del análisis de regresión lineal múltiple para las conductas consumo excesivo de comida y alcohol se presentan en la tabla 4. Un total de siete predictores explican la ingesta excesiva de comida durante el distanciamiento social por COVID-19
$\left(\mathrm{R}=.535 ; \mathrm{R}^{2}=.286 ; \mathrm{AR}^{2}=.281\right)$, mientras que seis predictores conforman el modelo para el consumo de alcohol durante el mismo periodo $\left(\mathrm{R}=.453 ; \mathrm{R}^{2}\right.$ $=.205 ; \mathrm{AR}^{2}=.200$ ).

Tabla 4. Modelos de regresión lineal múltiple para la ingesta excesiva de comida y consumo de alcohol durante el distanciamiento social por COVID-19

\begin{tabular}{lcccccc}
\hline & & COM & & & ALC & \\
& B & SE & $\beta$ & B & SE & B \\
Constante & -0.762 & 0.244 & & 6.361 & 0.752 & \\
EST & 0.089 & 0.01 & $0.265^{* * *}$ & 0.328 & 0.032 & $0.299^{* * *}$ \\
AF CONEG & 0.123 & 0.015 & $0.225^{* * *}$ & & & \\
ANS & 0.136 & 0.026 & $0.147^{* * *}$ & 0.276 & 0.053 & $0.148^{* * *}$ \\
SXMUJER & 0.542 & 0.13 & $0.111^{* * *}$ & -1.591 & 0.266 & $-0.162^{* * *}$ \\
APO & -0.047 & 0.016 & $-0.077^{* *}$ & & & \\
DXCOVIDOT & 0.373 & 0.128 & $0.075^{* *}$ & & & \\
RINT & 0.506 & 0.199 & $0.065^{*}$ & & & \\
EDA & & & & -0.079 & 0.015 & $-0.188^{* * *}$ \\
OCUEST & & & & -1.205 & 0.328 & $-0.126^{* * *}$ \\
AF DIST & & & & -0.127 & 0.037 & $-0.093^{* * *}$ \\
\hline
\end{tabular}

Nota. COVID-19 = Coronavirus disease. COM = Ingesta excesiva de comida; ALC = Consumo de alcohol; EST = Estrés psicológico; AF CONEG = Afrontamiento conductual (conductas negativas); ANS = Ansiedad fisiológica; SEXMUJER = Sexo mujer; APO = Red de apoyo social; DXCOVISOT = Diagnóstico de COVID-19 en familiares que comparten el hogar; RINT = Residencia en el interior de la República; EDA = Edad; OCUEST = Ocupación estudiante; AF DIST $=$ Estilo de afrontamiento distracción.

${ }^{*} \mathrm{p}<.05$

${ }^{* *} \mathrm{p}<.01$

${ }^{* * *} \mathrm{p}<.001$

18 | Ciencia y Sociedad 2021; 46(2, abril-junio): 7-30 • Artículos 


\section{Conducta alimentaria y consumo de alcohol durante el distanciamiento social por COVID-19 en México: un estudio exploratorio}

Finalmente, la figura 1 presenta, de manera gráfica, en comer y beber alcohol durante el distanciamiento el modelo predictivo para los excesos conductuales físico por COVID-19.

Figura 1. Modelo predictivo de la ingesta excesiva de comida y consumo de alcohol durante el distanciamiento social por COVID-19

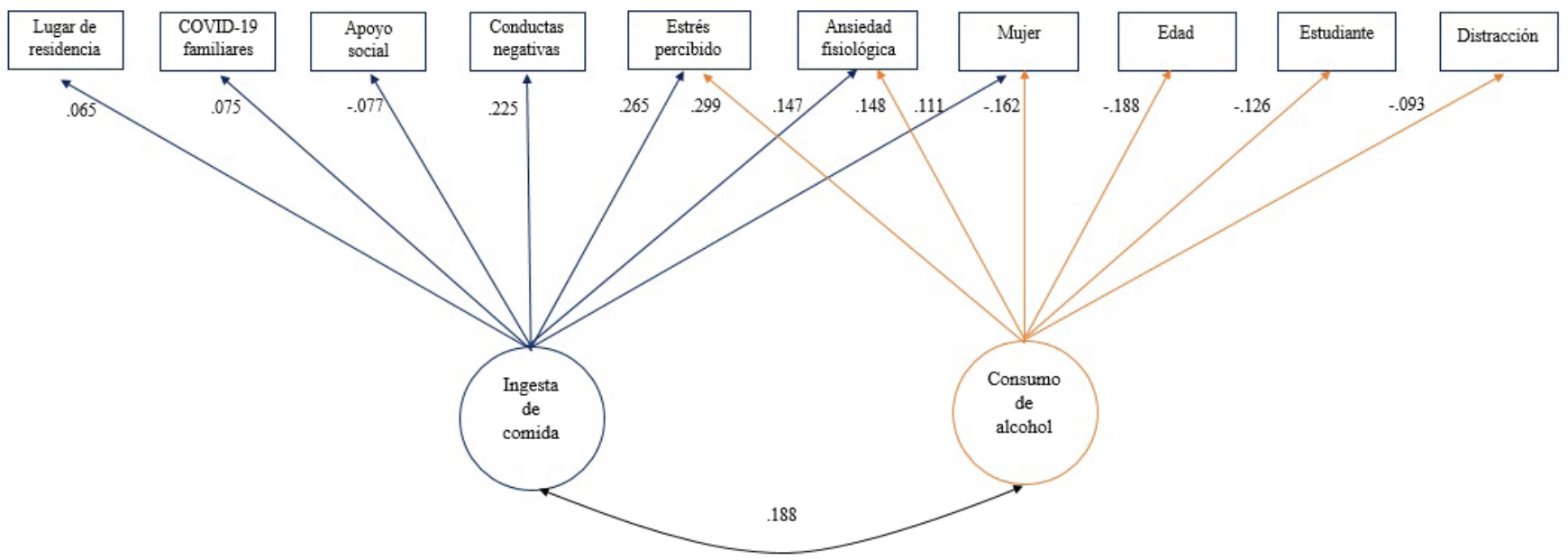

Nota. COVID-19 = Coronavirus disease. Lugar de residencia = lugar de residencia en el interior de la República; COVID-19 familiares = diagnóstico de COVID-10 en familiares que comparten el hogar; Apoyo social = red de apoyo social; Conductas negativas y Distracción se refieren a tipos de afrontamiento.

\section{Discusión}

El objetivo del estudio fue explorar la conducta alimentaria y el consumo de alcohol durante el distanciamiento social por COVID-19 e identificar las variables psicosociales asociadas a dichas conductas. La falta de consenso caracteriza a la investigación sobre predictores de la ingesta de comida y consumo de alcohol como forma de afrontamiento (Laitinen et al. 2002); más aún en una situación crítica y en constante evolución como la pandemia actual (Mayasari et al., 2020; Villanueva et al., 2021). Sin embargo, se considera que la principal aportación de los resultados del presente estudio es la identificación de factores psicosociales que pueden considerarse en el diseño de intervenciones en salud (Barbosa et al., 2020a, b; Rehm et al., 2020), ya que guardan estrecha relación con resultados obtenidos en investigaciones previas.
El aumento en el consumo de comida poco saludable y la disminución de la actividad física durante el distanciamiento social referidos por la muestra mexicana son parecidos a los reportados para poblaciones asiáticas, africanas y europeas estudiadas por Al-Musharaf (2020) y Ammar et al. (2020). No obstante, difieren de lo encontrado por Di Renzo et al. (2020) que hallaron mayor aumento en la actividad física (italianos $=38.6 \%$ vs. mexicanos $=29.2 \%$ ). Asimismo, la percepción de ganancia de peso difiere ligeramente de los hallazgos de Di Renzo et al. (2020) y Villanueva et al. (2020) (italianos $=48.6 \%$ vs. españoles $=49.8 \%$ vs. mexicanos $=41.5 \%$ ). Sobre la ingesta excesiva de comida durante el distanciamiento físico los encuestados mexicanos mostraron mayor probabilidad de desarrollar un trastorno por atracón (36.3 \%) que los encuestados brasileños (22.8 \%) del estudio de Hanada et al. (2020). 
El modelo predictivo obtenido para la ingesta excesiva de comida coincide en dos factores con el modelo presentado por Haddad et al. (2020), a saber, sexo femenino (libaneses: $\beta=.52$ vs mexicanos $\beta=.111$ ) y ansiedad (libaneses: $\beta=.04$ vs mexicanos $\beta=.147$ ). A su vez, la asociación entre sexo femenino y alteraciones en el apetito también es mencionada por el modelo predictivo de Di Renzo et al. (2020). Si bien el estrés no es referido como variable asociada a la ingesta de comida, la relación está documentada en la literatura (Al-Musharaf, 2020; Laitinen et al., 2002). De forma similar, los modelos previos no encontraron el apoyo social como factor protector ante la ingesta excesiva de comida. El hecho de que se hallara en la presente investigación se puede explicar a partir de lo que sostienen Laitinen et al. (2002) sobre el apoyo como recurso de afrontamiento en situaciones estresantes, el cual, según estos autores, brinda contención emocional y disminuye la búsqueda de actividades de desahogo emocional como comer. Cabe señalar que los modelos derivados de los estudios previos tampoco han considerado variables como la convivencia con un familiar con diagnóstico de COVID-19, por supuesto, se debe tener en cuenta que la muestra mexicana contemplada refirió la posibilidad del contagio y muerte de un familiar en periodo de pandemia como el estresor más relevante durante el distanciamiento social. Igualmente, el estilo de afrontamiento no se menciona en otros estudios, por tanto, su investigación constituye una nueva y fructífera línea, ya que su asociación con el estrés y las conductas excesivas de comer y beber está documentada (Laitinen et al., 2002). De igual modo, es necesario estudiar la asociación entre las variables lugar de residencia y conducta alimentaria, ya que en el estudio de López et al. (2020) también resultó significativa, pero solo en cuanto a la ganancia de peso.

Para el consumo de alcohol, al igual que las muestras consideradas por Ammar et al. (2020), el Canadian
Centre on Substance Use and Addiction (2020) y Villanueva et al., (2020), la muestra mexicana percibió una disminución en el uso de bebidas alcohólicas. Sin embargo, los participantes mexicanos percibieron una reducción mayor (canadienses $=10 \%$ vs. españoles $=15,1 \%$ vs mexicanos = $45.9 \%)$. Asimismo, a diferencia de los reportado por APA (2021), y Barbosa et al. (2020a, 2020b), el aumento en el consumo de alcohol en México solo fue del $9.3 \%$ vs. $31 \%$ reportado por las muestras estadounidenses. Probablemente, la disminución percibida por parte de los participantes mexicanos se deba a las políticas de restricción aplicadas a la industria del alcohol durante la fase de la pandemia evaluada en esta investigación, así como la puesta en marcha de la llamada ley seca o prohibición de venta de bebidas con graduación alcohólica durante la Fase 3 de la pandemia. Aunque también puede estar relacionado con las características de la muestra, ya que la mayoría refirió un consumo responsable e incluso la no ingesta de alcohol. No obstante, se sugiere realizar más estudios debido a que Natera (2020) reportó aumento en el consumo de alcohol para una muestra mexicana.

En cuanto al modelo predictivo obtenido, los resultados del presente estudio coinciden con los reportados por Capasso et al. (2021) en lo que respecta a ansiedad (estadounidense: $\mathrm{OR}=1.41 ; 95 \% \mathrm{IC}$ $=1.20-1.66 ; \mathrm{p}<.05$ vs. mexicanos: $\mathrm{B}=.276 ; \mathrm{SE}=$ $.053 ; \beta=.148 ; \mathrm{p}<.001$ ) y edad (estadounidenses: $\mathrm{OR}=.73 ; 95 \% \mathrm{IC}=.63, .83 ; \mathrm{p}<.05$ vs. mexicanos: $\mathrm{B}=-.079 ; \mathrm{SE}=.015 ; \beta=-.188 ; \mathrm{p}<.001)$. El que el sexo femenino constituya un factor de protección en el modelo presentado difiere de lo encontrado por Barbosa et al. (2020 a, b) y Graupensperger et al. (2021), pero puede estar relacionado con el hecho de que las mujeres muestran puntuaciones altas para el consumo moderado de alcohol, pero las puntuaciones disminuyen a medida que aumenta el número de tragos o consumo de riesgo (Canadian Centre on Substance Use and Addiction, 2020; Natera, 2020; 


\section{Conducta alimentaria y consumo de alcohol durante el distanciamiento social por COVID-19 en México: un estudio exploratorio}

Rodríguez et al., 2020; Villanueva et al., 2021). Igualmente, el que la ocupación estudiante en la muestra mexicana sea un factor protector para el consumo de alcohol durante el distanciamiento social puede estar asociado a los hallazgos de Graupensperger et al. (2021) y Villanueva et al. (2021) quienes encontraron que los estudiantes que viven con sus padres disminuyeron su consumo. Lo anterior puede estar relacionado con el hecho de que esta población tiende a consumir bebidas alcohólicas en lugares que se encontraban cerrados en el momento del estudio como parte de las medidas de control del brote. Por otro lado, la identificación del estilo de afrontamiento distracción como factor protector para el consumo de alcohol durante el distanciamiento social revela, de forma indirecta, que el aburrimiento por estar en casa sin realizar actividades distractoras influye en la práctica de conductas poco saludables (Brooks et al., 2020; CONADIC, 2020).

Por último, se puntualizan las principales limitaciones metodológicas del estudio; estas tienen que ver con el tipo de diseño, su alcance, muestreo e instrumentos empleados. Debido al momento y forma en que se recolectaron los datos, a la falta de investigaciones locales similares, el carácter exploratorio y transversal de la investigación, la sobre representación de algunos grupos poblacionales, el uso de instrumentos de autoinforme en lugar de la observación directa de conductas y la pandemia como evento en constante transformación resulta imposible realizar generalizaciones, ya que las limitaciones señaladas favorecen la descripción de la muestra y no necesariamente de la población mexicana. Empero, la metodología y resultados obtenidos contribuyen a la generación de hipótesis e incentiva la investigación futura, para la cual se sugiere el empleo de muestras probabilísticas, la inclusión de grupos vulnerables que muestran dificultades para acceder a encuestas en línea, la medición por observación directa, registros y autoinformes de otras variables relevantes para las conductas de interés y la preferencia por diseños longitudinales.

\section{Conclusiones}

Las limitaciones de la investigación no restan valor a sus aportaciones en materia de la identificación de factores psicológicos y sociales que se relacionan con conductas de riesgo como el comer y beber en exceso durante el distanciamiento social por COVID-19. Con base en los resultados se propone una estrategia de intervención psicosocial que incluya: 1) psicoeducación sobre los efectos en la salud física, emocional, psicológica y social del consumo excesivo de comida y alcohol; 2) desmitificación de creencias sobre los alimentos, el alcohol y COVID$19 ; 3)$ técnicas de desactivación fisiológica para el manejo de los síntomas somáticos de ansiedad y estrés; 4) activación conductual con el propósito de disminuir los periodos de aburrimiento a partir de actividades planificadas y valiosas para la vida; 5) entrenamiento en solución de problemas para favorecer los estilos de afrontamiento orientados a la aproximación; 6) entrenamiento en autocontrol, con la finalidad de contribuir a la disminución de conductas perjudiciales para la salud y 7) auto reforzamiento, para que la persona sea capaz, por sí misma, de mantener los cambios generados. Además, es fundamental que las intervenciones se diseñen en formatos que aseguren el acceso y disponibilidad en función de las medidas sanitarias vigentes, así como de las condiciones económicas y tecnológicas de la población. Es decir, mientras las intervenciones presenciales no sean una alternativa viable, se deberán elegir otros medios de provisión, por ejemplo, carta, radio, televisión, teléfono e internet. Asimismo, es importante que cuenten con perspectiva de género y respeto a la diversidad de la población mexicana. De esta forma, se podrá contribuir a la mitigación de los efectos biopsicosociales de las medidas de control del actual brote epidémico y otras crisis de salud y desastres que pudieran presentarse. 


\section{Referencias}

Al-Musharaf, S. (2020). Prevalence and Predictors of Emotional Eating among Healthy Young Saudi Women during the COVID-19 Pandemic. Nutrients, 12(10), 2923. https:// doi.org/10.3390/nu12102923

American Psychological Association. (2021). Drinking, coping, and COVID-19. https://www. apa.org/monitor/2021/01/alcohol-covid

Ammar, A., Brach, M., Trabelsi, K., Chtourou, H., Boukhris, O., Masmoudi, L., Bouaziz, B., Bentlage, E., How, D., Ahmed, M., Müller, P., Müller, N., Aloui, A., Hammouda, O., Paineiras-Domingos, L. L., Braakman-jansen, A., Wrede, C., Bastoni, S., Pernambuco, C. S... Hoekelmann, A. (2020). Effects of COVID-19 Home Confinement on Eating Behaviour and Physical Activity: Results of the ECLB-COVID19 International Online Survey. Nutrients, 12(1583). https://doi. org/10.3390/nu12061583

Armitage, R., \& Nellums, L. B. (2020). COVID-19 and the consequences of isolating the elderly. Lancet Public Health, 5(5). https://doi.org/ 10.1016/S2468-2667(20)30061-X

Babor, T. F., Higgins-Biddle, J. C., Saunders, J. B., \& Monteiro, M. G. (2001). AUDIT. Cuestionario de Identificación de los Trastornos debidos al Consumo de Alcohol. Pautas para su utilización en Atención Primaria. Organización Mundial de la Salud. https://www.who.int/ substance_abuse/activities/en/AUDITmanualSpanish.pdf

Barbosa, C., Cowell, A.J., \& Dowd, W.N. (2020a). Alcohol Consumption in Response to the COVID-19 Pandemic in the United States. Journal of Addiction Medicine. https://doi.org/ 10.1097/ADM.0000000000000767

Barbosa, C., Cowell, A. J., \& Dowd, W. N. (2020b). How Has Drinking Behavior Changed During the COVID-19 Pandemic? [Diapositiva de PowerPoint]. Research Triangle Institute Inter- national. https://www.rti.org/sites/default/ files/covid19_alcohol_survey_webinar_ slides_071420.pdf

Brooks, S. K., Webster, R. K., Smith, L. E., Woodland, L., Wessely, S., Greenberg, N., \& Rubin, G.J. (2020). The psychological impact of quarantine and how to reduce it: rapid review of the evidence. Lancet, 395(10227), 912-920. https://doi.org/10.1016/S01406736(20)30460-8

Canadian Centre on Substance Use and Addiction. (2020). Boredom and Stress Drives Increased Alcohol Consumption during COVID-19: NANOS Poll Summary Report. https://www. ccsa.ca/boredom-and-stress-drives-increased-alcohol-consumption-during-covid-19-nanos-poll-summary-report

Capasso, A., Jones, A. M., Ali, S. H., Foreman, J., Tozan, Y., \& DiClemente, R. J. (2021). Increased alcohol use during the COVID-19 pandemic: The effect of mental health and age in a cross-sectional sample of social media users in the U.S. Preventive Medicine, 145(106422). https://doi.org/10.1016/j. ypmed.2021.106422

Carrillo-Valdez, L. (2020). Psychometric properties of the Coronavirus Anxiety Scale in Mexican adult population. Ciencia y Humanismo en la Salud, 7(3), 45-60. http://revista.medicina. uady. $\mathrm{mx} / \mathrm{revista} /$ index.php/cienciayhumanismo/article/view/150

Clay, J. M. \& Parker, M. O. (2020). Alcohol use and misuse during the COVID-19 pandemic: a potential public health crisis? Lancet Public Health, 5(5). https://doi.org/10.1016/S24682667(20)30088-8

Comisión Nacional contra las Adicciones. Secretaría de Salud. (2020). Consumo de bebidas alcohólicas durante la Jornada Nacional de Sana Distancia por COVID-19. https://www.gob.mx/salud/ conadic/documentos/consumo-de-bebidas-alcoholicas-durante-la-jornada-nacional-de-sana-distancia-por-covid-19 
Di Renzo, L., Gualtieri, P., Pivari, F., Soldati, L., Attinà, A., Cinelli, G., Leggeri, C., Caparello, G., Barrea, L., Scerbo, F., Esposito, E., \& De Lorenzo, A. (2020). Eating habits and lifestyle changes during COVID-19 lockdown: an Italian survey. Journal of Translational Medicine, 18(229). https://doi.org/10.1186/ s12967-020-02399-5

Feldman, R. S. (2007). Desarrollo psicológico a través de la vida. Pearson Educación. http:// up-rid2.up.ac.pa:8080/xmlui/handle/ $123456789 / 1406$

Grau, L. (2014). Test de Trastorno por Atracón. https://www.studocu.com/pe/document/ universidad-ricardo-palma/fisica/practica/test-de-trastorno-por-atracon-pagina-web/6558364/view

Graupensperger, S., Jaffe, A. E., Fleming, C. N. B., Kilmer, J. R., Lee, C. M., \& Larimer, M. E. (2021). Changes in College Student Alcohol Use During the COVID-19 Pandemic: Are Perceived Drinking Norms Still Relevant? Emerging Adulthood, 1-10. https://doi. org/10.1177/2167696820986742

Haddad, C., Zakhour, M., Boukheir, M., Haddad, R., Al Hachach, M., Sacre, H., \& Salameh, P. (2020). Association between eating behavior and quarantine/confinement stressors during the coronavirus disease 2019 outbreak. Journal of Eating Disorders, 8(40). https:// doi.org/10.1186/s40337-020-00317-0

Hanada, T., Cestari, R., Miguel, L. E., Miguel, B., Casati, M, \& Pereida, R. (2020) Periodic Binge Eating Disorder during the Social Isolation Due to Covid-19 Pandemic. Journal of Obesity and Eating Disorders, 6(4). https://doi. org/10.36648/2471-8203.6.4.100059

Higareda, J. J., Del Castillo, A., Romero, A., Gil, F. M. E., \& Rivera, S. (2015). La escala de estilos de afrontamiento forma BMOOS: validación en estudiantes universitarios mexicanos. Psicología Iberoamericana, 23(2), 55-65. http:// revistas.ibero.mx/psicologia/articulo_detalle. php?id_volumen=13\&id_articulo=140\&id_ seccion $=\&$ active $=1$ \&pagina $=1$

Laitinen, J., Ek, E., \& Sovio, U. (2002). Stress-Related Eating and Drinking Behavior and Body Mass Index and Predictors of This Behavior. Preventive Medicine, 34, 29-39. https://doi. org/10.1006/pmed.2001.0948

Lazarus, R. S. \& Folkman, S. (1984). Stress, appraisal and coping. Springer.

López, M., Bellido, D., Monereo, S., Lecube, A., Sánchez, E., \& Tinahones, F. J. (2020). Ganancia de peso durante el confinamiento por la COVID-19; encuesta de la Sociedad Española de Obesidad. Revista de la Sociedad Española de Cirugía de Obesidad y Metabólica y de la Sociedad Española para el Estudio de la Obesidad, 10(2). https://www.bmi-journal. com/index.php/bmi/article/view/739

Mayasari, N. R., Ho, D. K. N., Lundy, D. J., Skalny, A. V., Tinkov, A. A., Teng, I., Wu, M., Faradina, A., Mohammed, A. Z. M., Park, J. M., Ngu, Y. J., Aliné, S. Shofia, N. M., \& Chang, J. (2020). Impacts of the COVID-19 Pandemic on Food Security and Diet-Related Lifestyle Behaviors: An Analytical Study of Google Trends-Based Query Volumes. Nutrients, 12(3103). https://doi.org/10.3390/ nu12103103

Moos, R. H. (2010). CRI-A. Inventario de Respuestas de Afrontamiento Adultos. TEA Ediciones.

Natera, G. (2020). La COVID, el alcohol y la depresión: ¿Qué pasó en México en el primer nivel de atención y las DIT, durante la pandemia? [Webinar]. Organización Panamericana de la Salud. https://www.paho.org/es/documentos/ covid-alcohol-depresion-que-paso-mexico-primer-nivel-atencion-dit-durante-pandemia

Ramalho, R. (2020). Alcohol consumption and alcohol-related problems during the COVID-19 pandemic: a narrative review. Australasian Psychiatry, 28(5) 524-526. https://doi.org/ $10.1177 / 1039856220943024$ 
Rehm, J., Kilian, C., Ferreira-Borges, C., Jernigan, D., Monteiro, M., Parry, C. D. H., Sanchez, Z. M., \& Manthey, J. (2020). Alcohol use in times of the COVID 19: Implications for monitoring and policy. Drug and Alcohol Review, 39, 301-304. https://doi.org/10.1111/dar.13074

Rodríguez, L. M., Litt, D. M., \& Stewart, H. (2020). Drinking to cope with the pandemic: The unique associations of COVID-19related perceived threat and psychological distress to drinking behaviors in American men and women. Addictive Behaviors, 110(106532). https://doi.org/10.1016/j. addbeh.2020.106532

Touyz, S., Lacey, H., \& Hay, P. (2020). Eating disorders in the time of COVID-19. Journal of Eating Disorders, 8(19). https://doi.org/ 10.1186/s40337-020-00295-3

Velasco, A. E. (2017). Estrés en la vivienda [Tesis de doctorado, Universidad Nacional Autónoma de México]. Repositorio de Tesis DGBSDI: TESIUNAM. http://132.248.9.195/ptd2017/ enero/0754641/Index.html

Villamil, V., Valencia, M., Medina-Mora, M. E., \& Juárez, F. (2009). Validación de la prueba de identificación de trastornos derivados del consumo de alcohol (AUDIT) en pacientes Mexicanos con esquizofrenia. Revista Panamericana de Salud Pública, 26(4), 283-289. http:// www.scielosp.org/scielo.php?script=sci_ arttext\&pid=S1020-49892009001000001
Villanueva, V. J, Motos, P., Isorna, M., Villanueva, V., Blay, P., \& Vázquez-Martínez, A. Impacto de las medidas de confinamiento durante la pandemia de Covid-19 en el consumo de riesgo de alcohol. Revista Española de Salud Pública, 95, e202101015. https://www.mscbs.gob.es/biblioPublic/ publicaciones/recursos_propios/resp/ revista_cdrom/VOL95/ORIGINALES/ RS95C_202101015.pdf

Zvolensky, M. J., Garey, L., Rogers, A. H., Schmidt, N. B., Vujanovic, A. A., Storch, E. A., Buckner, J. D., Paulus, D. J., Alfano, C., Smits, J. A. J., \& O’Cleirigh, C. (2020). Psychological, addictive, and health behavior implications of the COVID-19 Pandemic. Behaviour Research and Therapy, 134(103715). https://doi.org/10. 1016/j.brat.2020.103715 
Conducta alimentaria y consumo de alcohol durante el distanciamiento social por COVID-19 en México: un estudio exploratorio

Apéndice 1. Medias, diferencias y asociaciones de las respuestas de ansiedad, estrés, afrontamiento, ingesta excesiva de comida y consumo de alcohol durante el distanciamiento social por COVID-19 de acuerdo con variables sociodemográficas

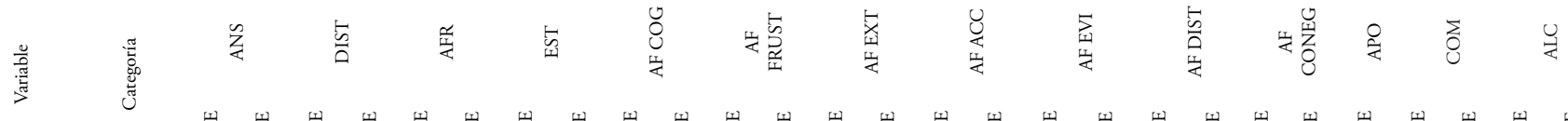

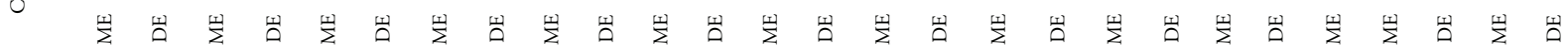

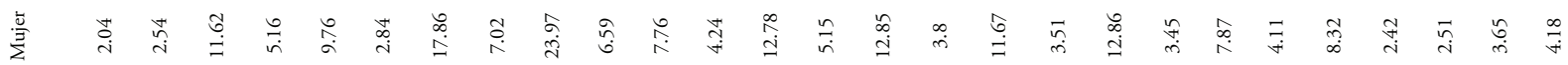

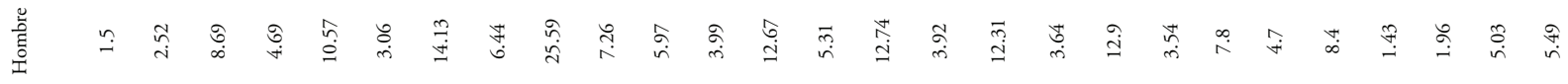

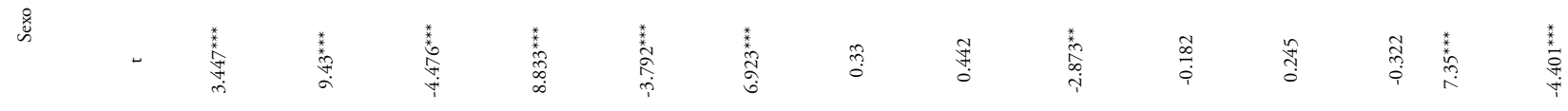

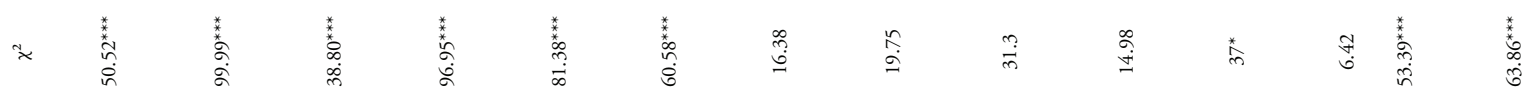

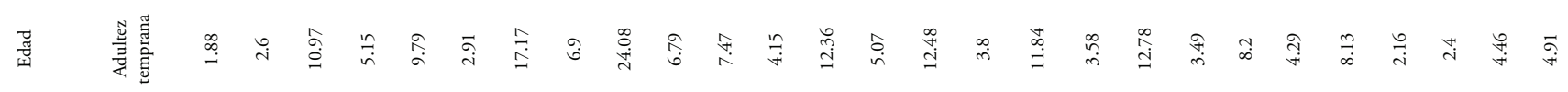

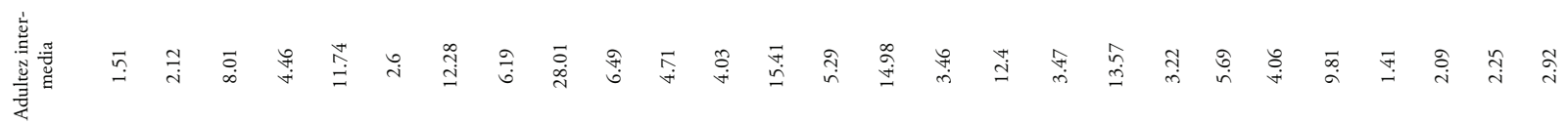

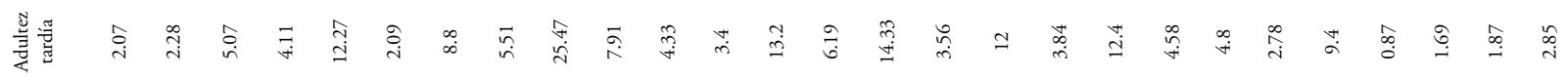

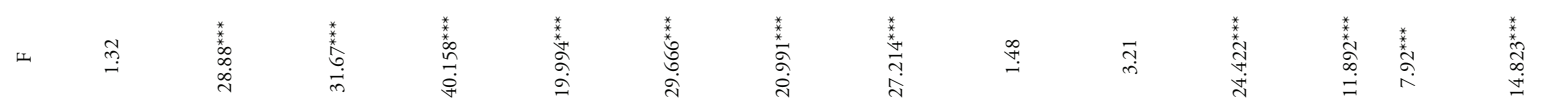

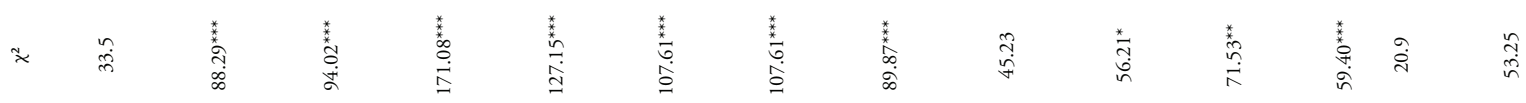

产

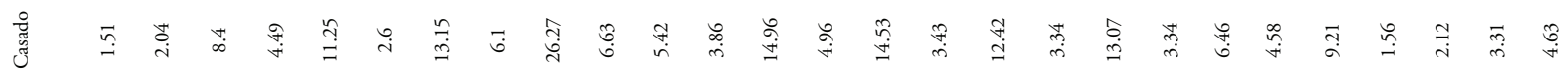

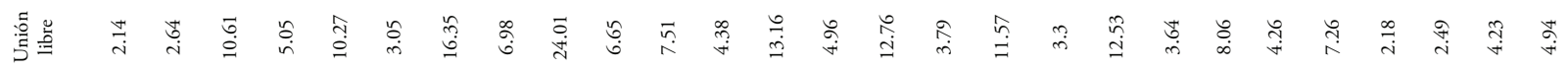

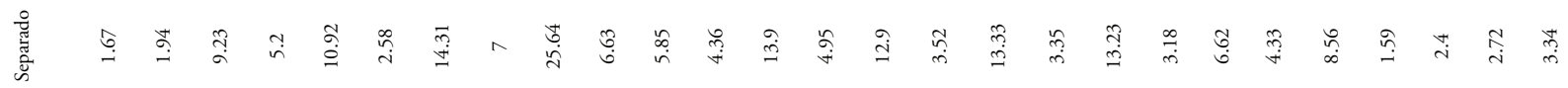

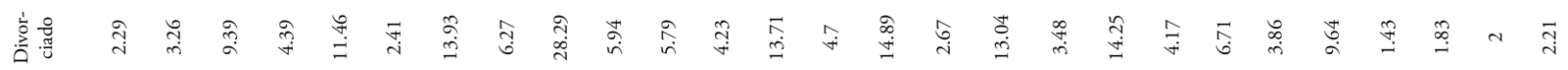

产

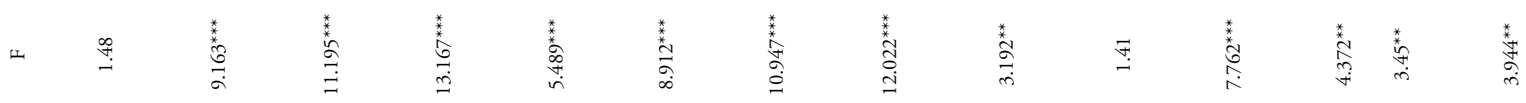

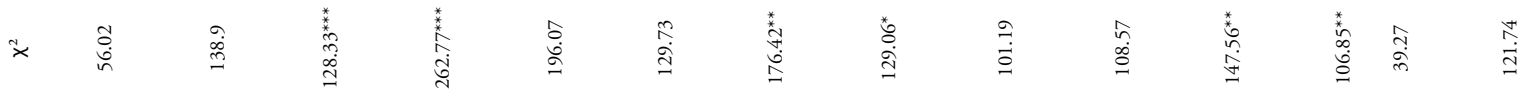




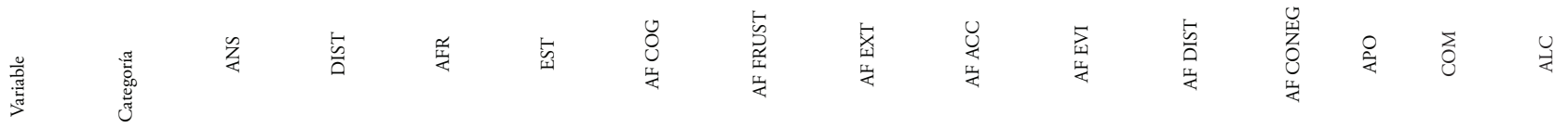

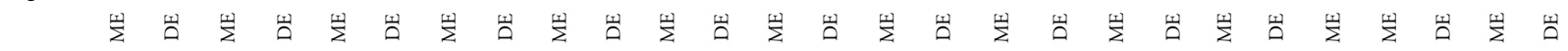

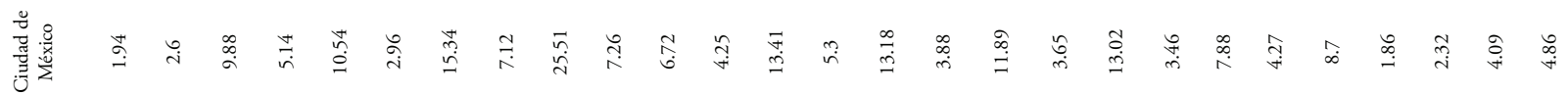

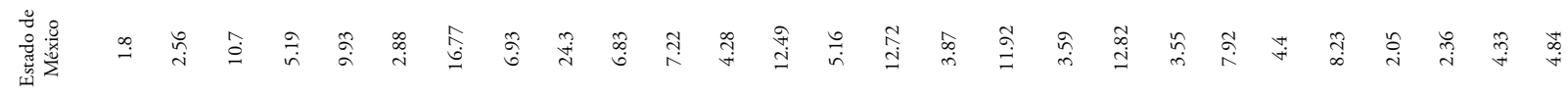

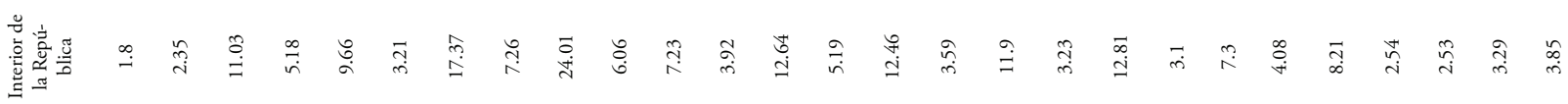

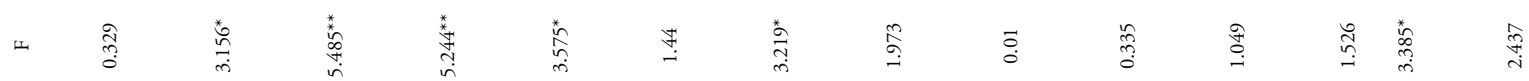

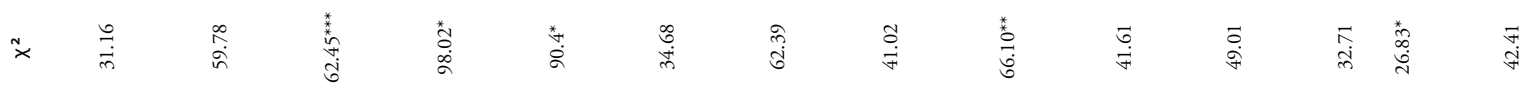

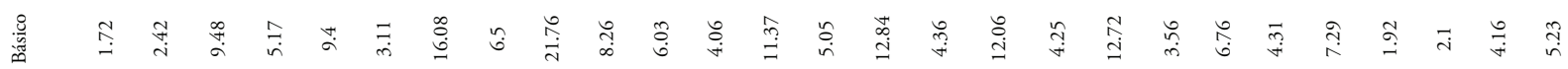

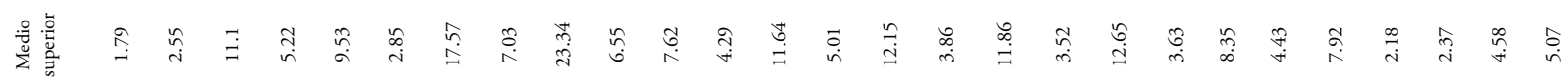

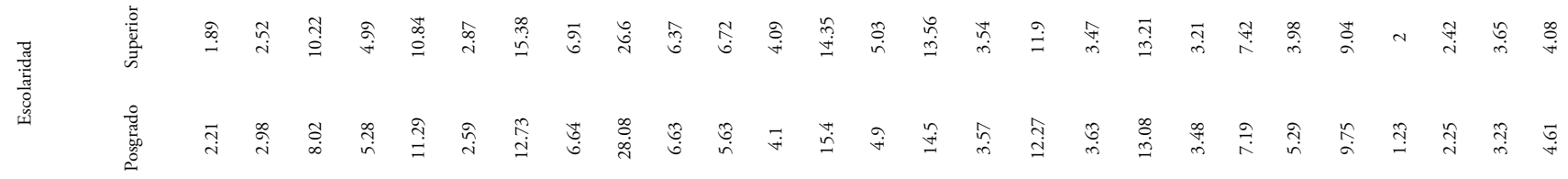

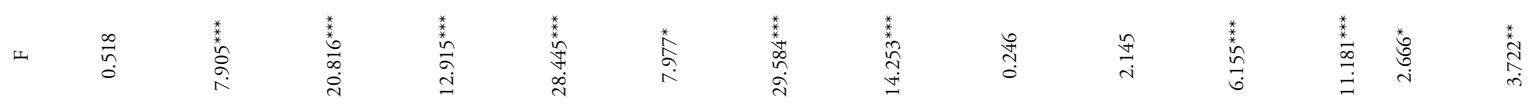

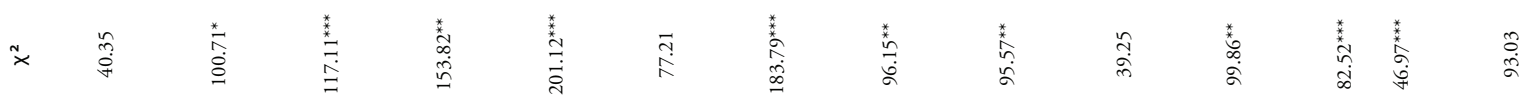

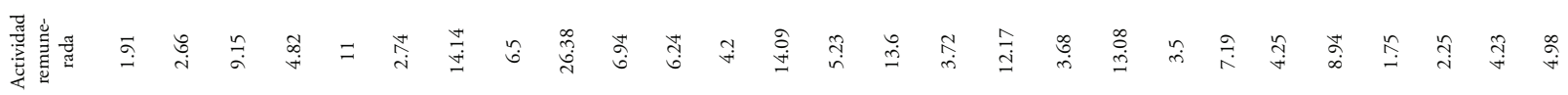

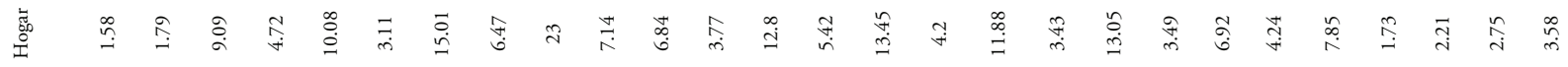

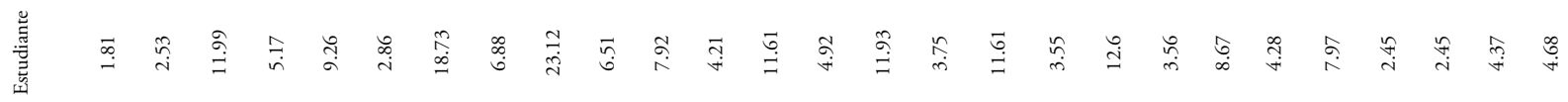

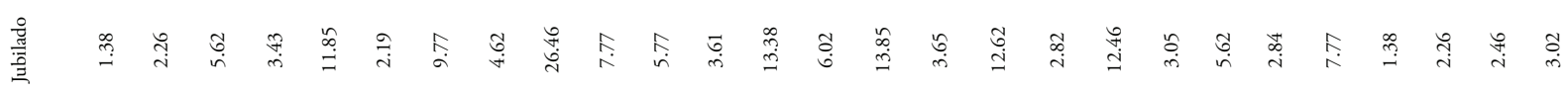

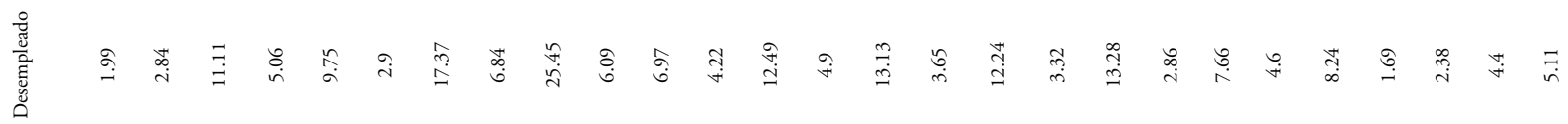

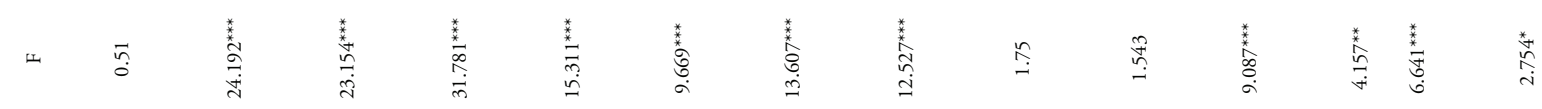

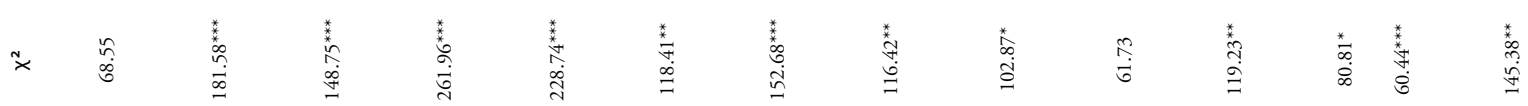




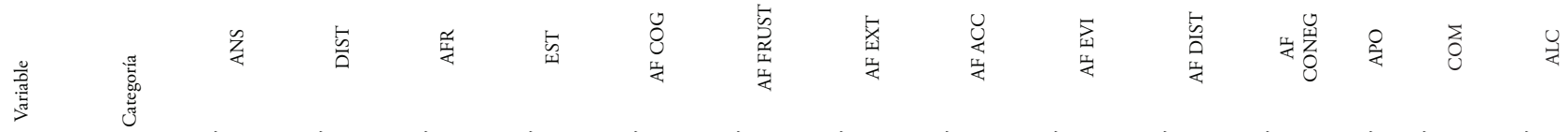

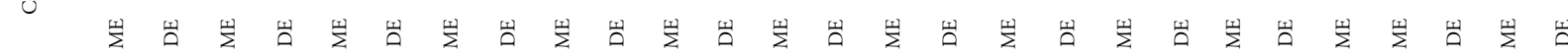

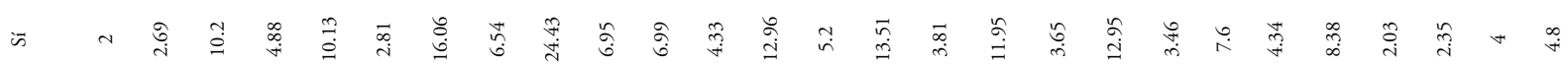

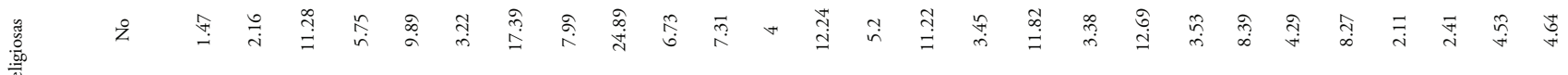

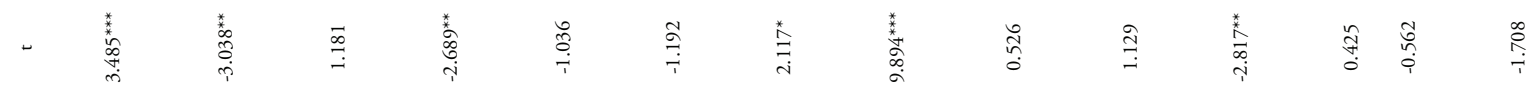

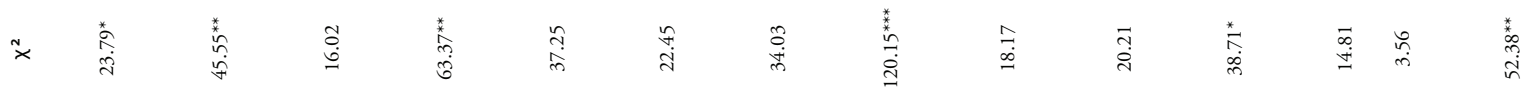

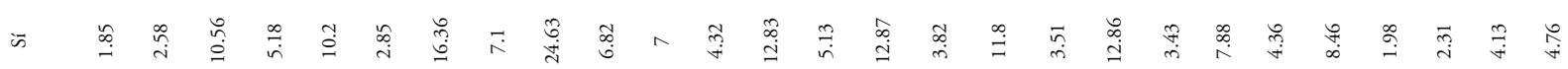

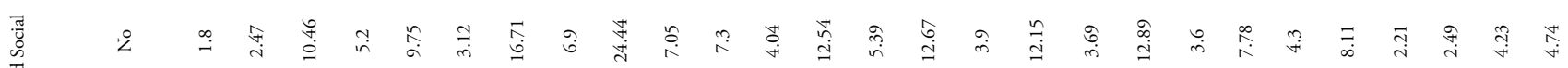

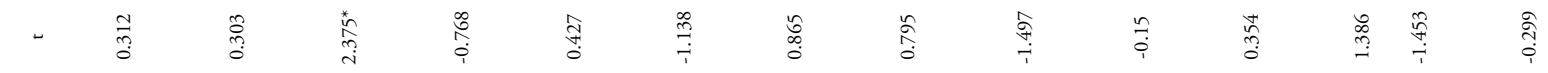

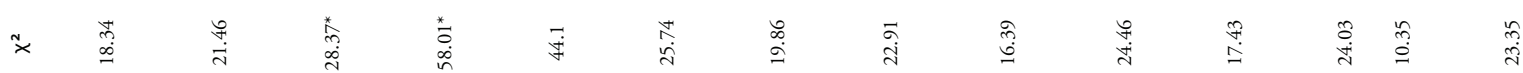

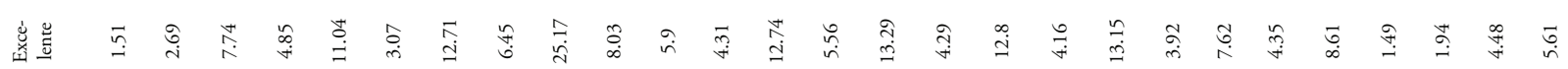

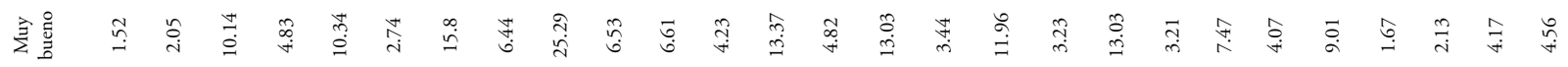

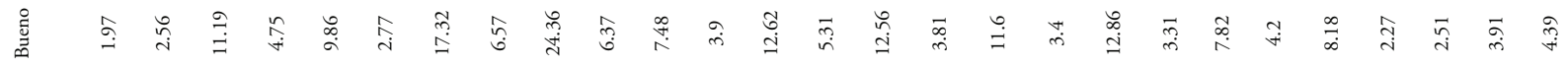

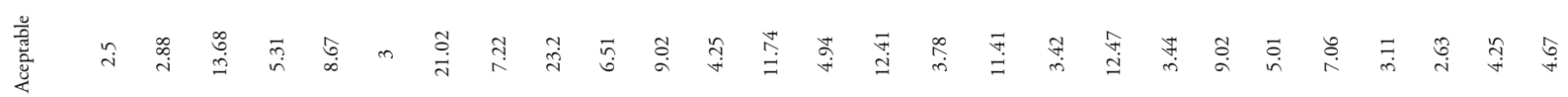

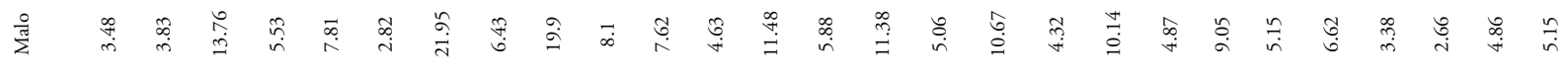

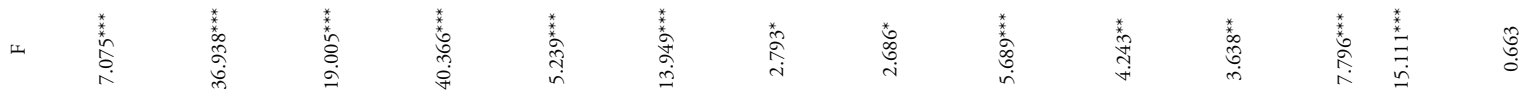

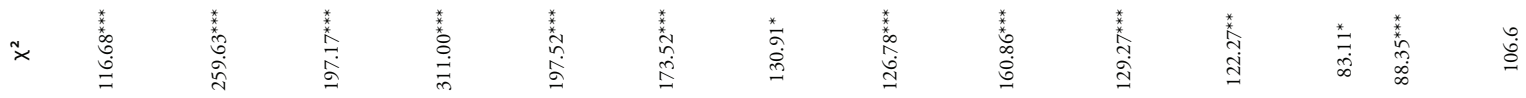

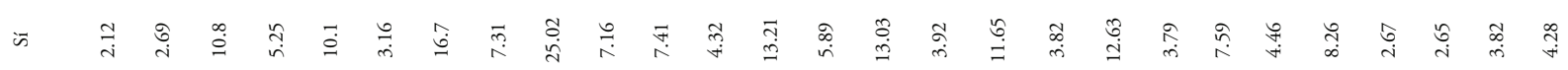

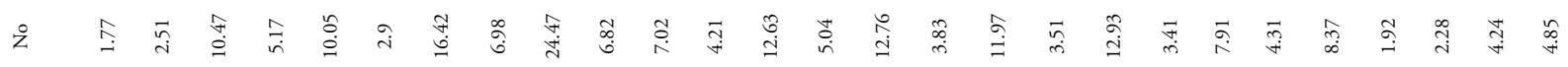

-

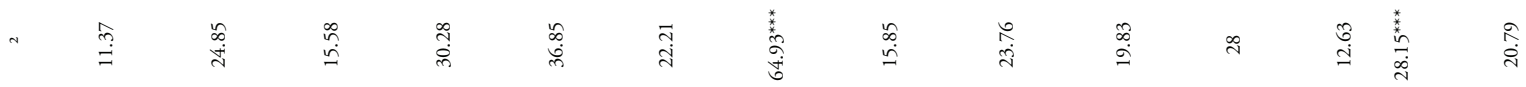




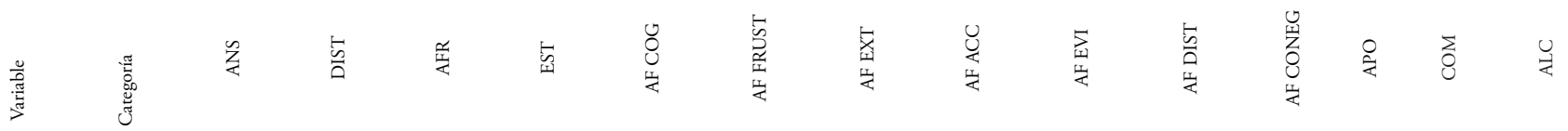

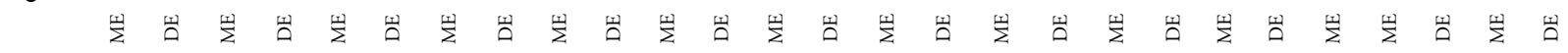

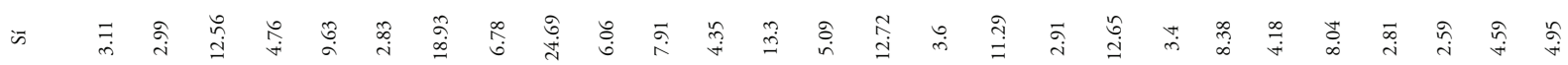

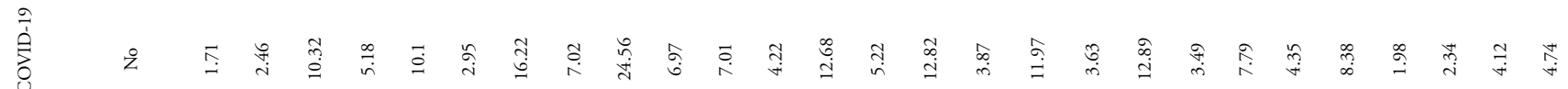

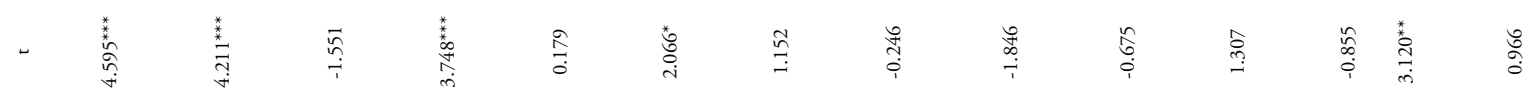

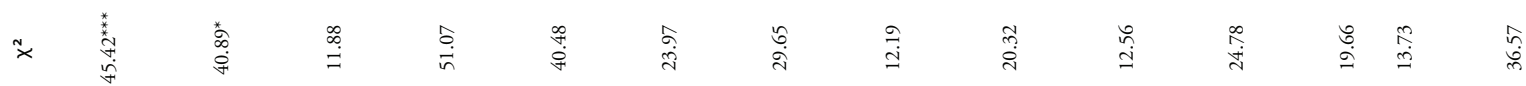

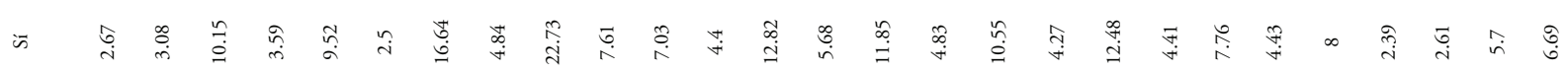

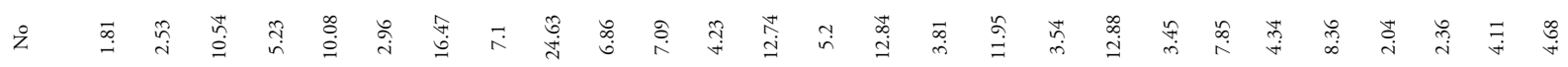

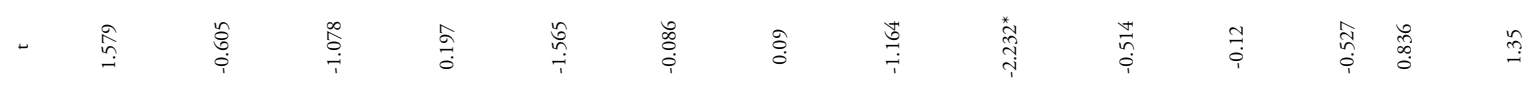

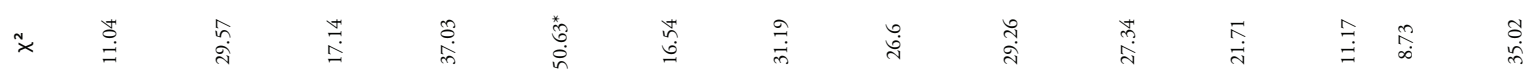

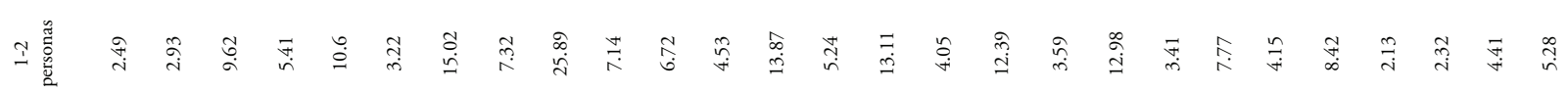

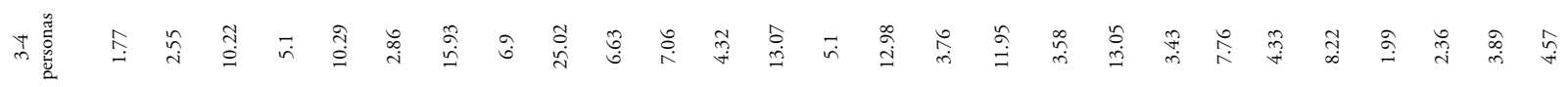

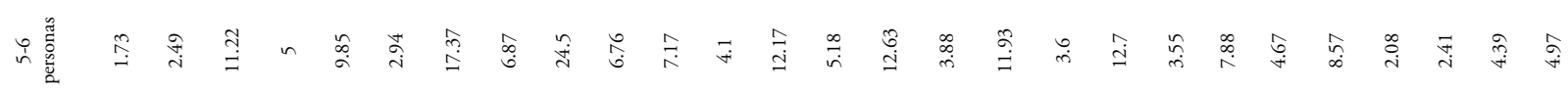

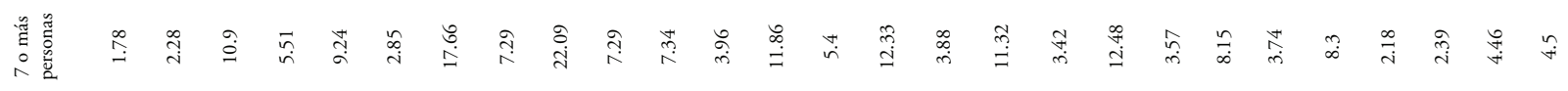

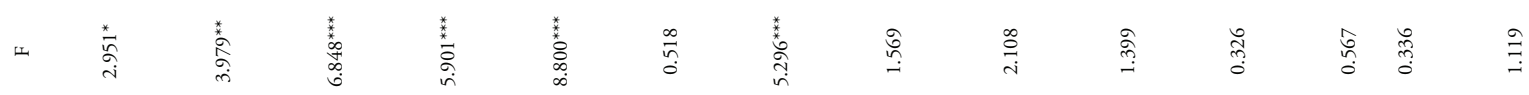

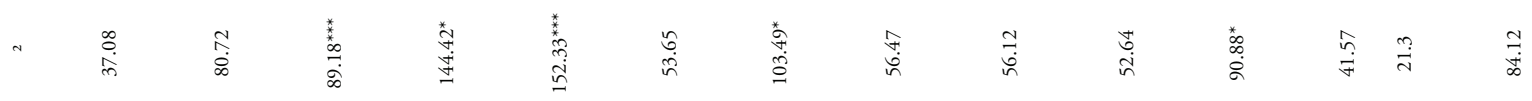

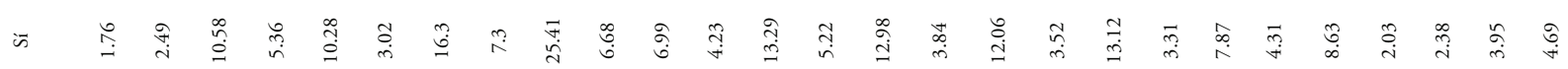

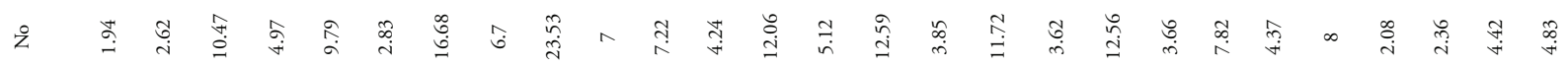

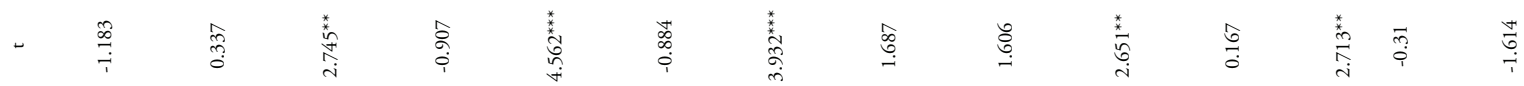

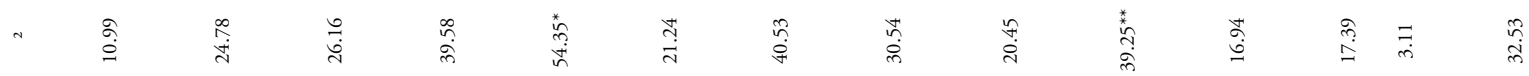




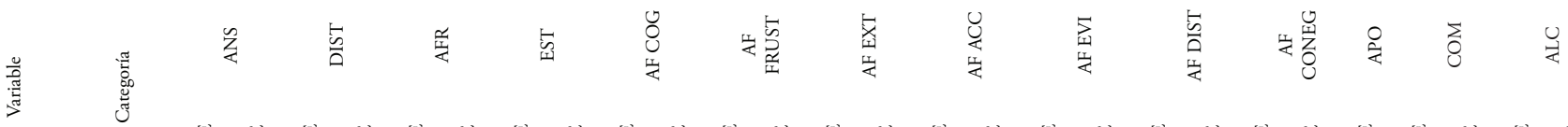

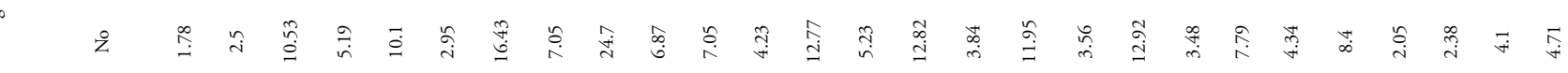

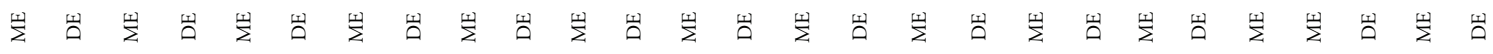
क

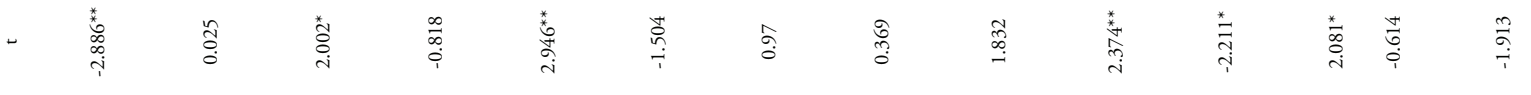

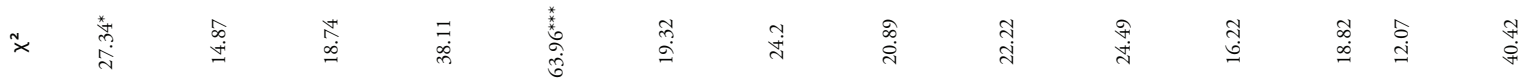

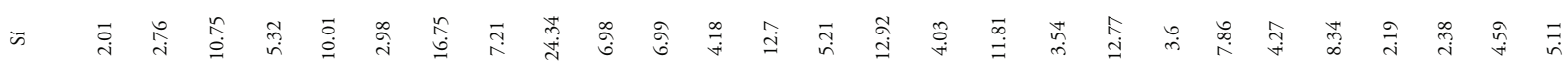

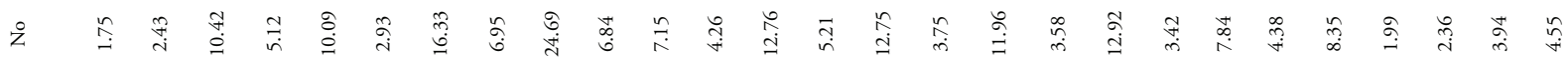

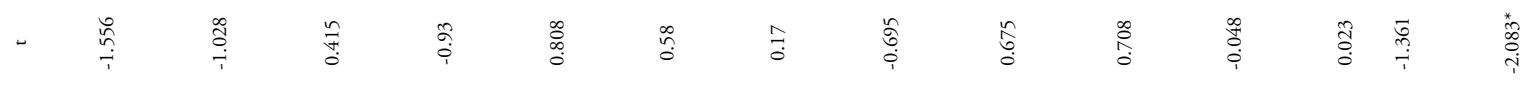

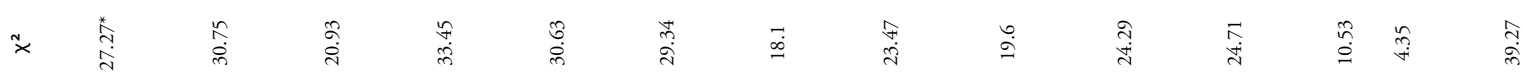

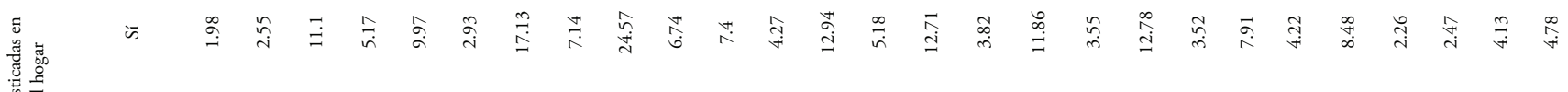

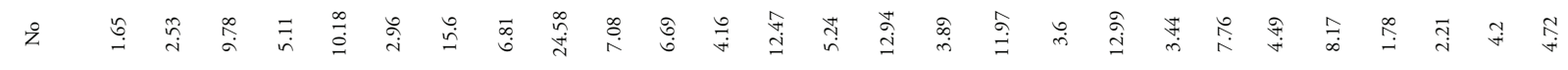

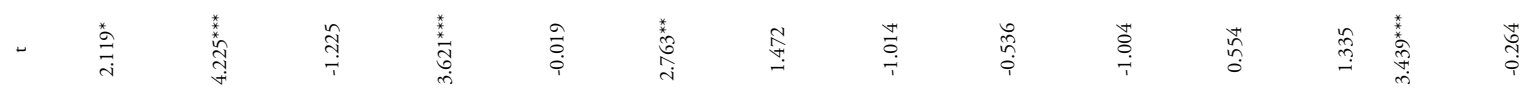

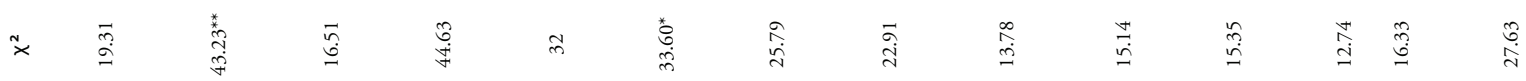

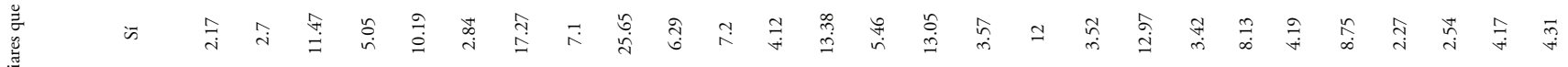

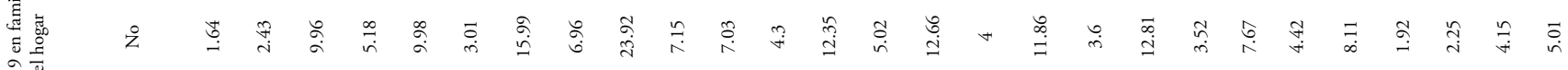

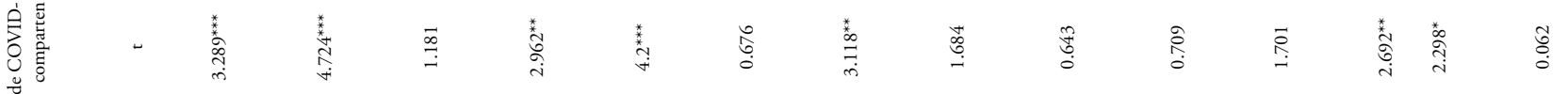
×

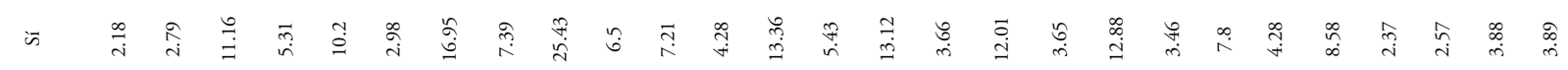

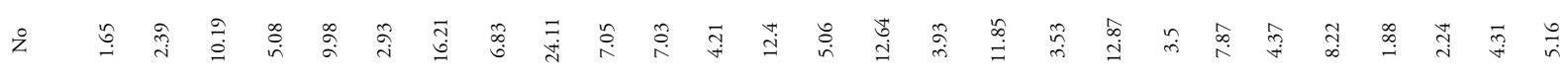

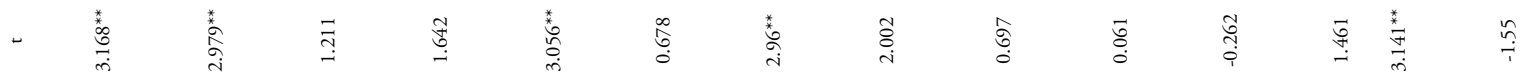

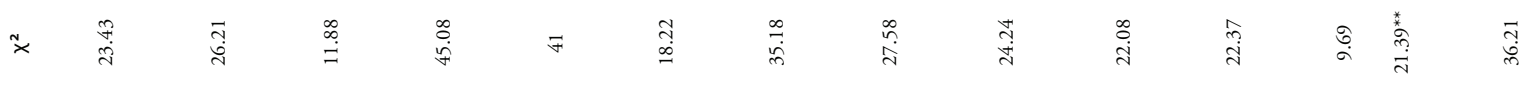




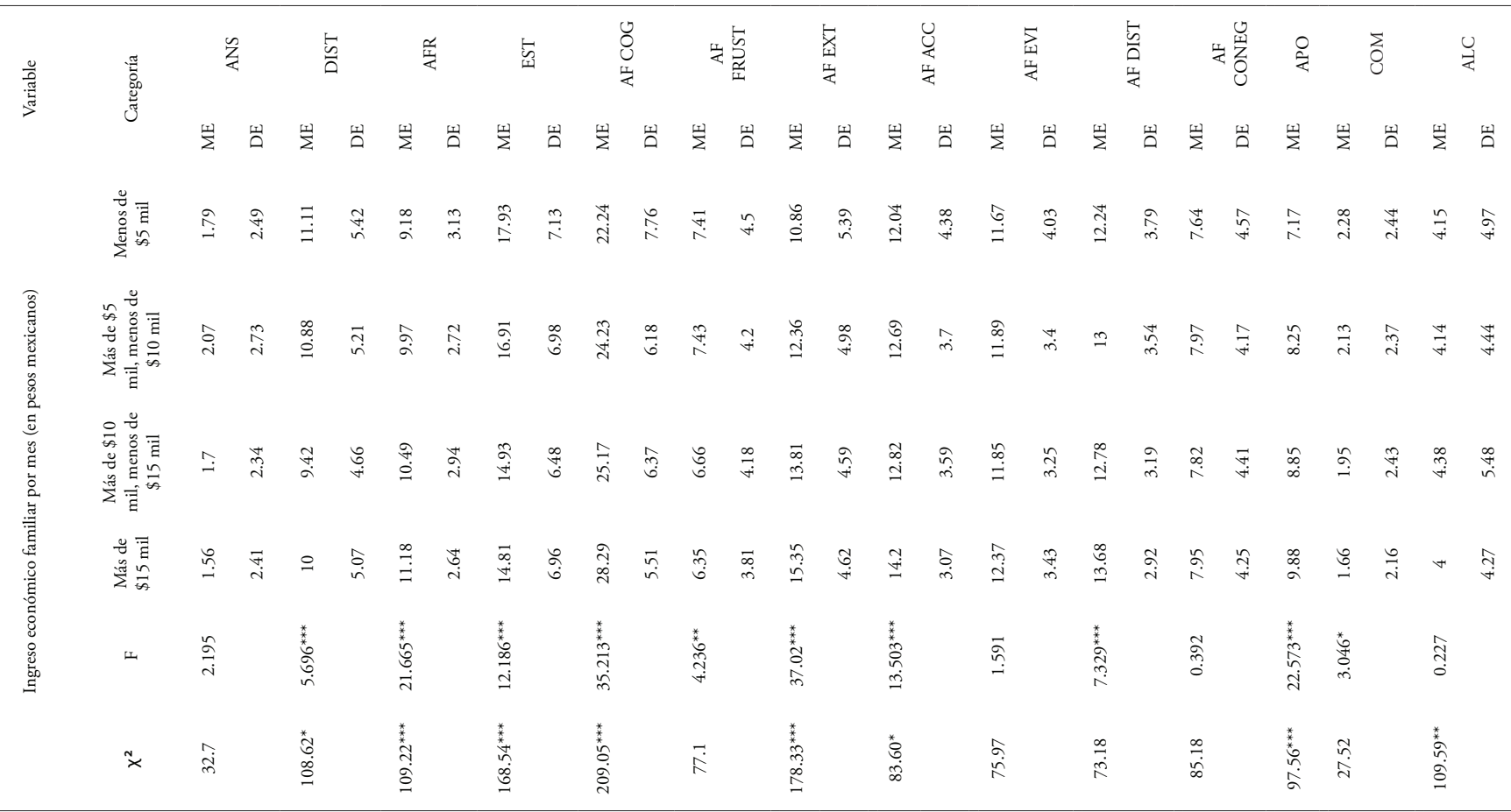

Nota. COVID-19 = Coronavirus disease. COM = Ingesta excesiva de comida; ALC = Consumo de alcohol; EST = Estrés psicológico; AF CONEG = Afrontamiento conductual (conductas negativas); ANS = Ansiedad fisiológica; SEXMUJER = Sexo mujer; APO = Red de apoyo social; DXCOVISOT = Diagnóstico de COVID-19 en familiares que comparten el hogar; RINT = Residencia en el interior de la República; EDA = Edad; OCUEST = Ocupación estudiante; AF DIST = Estilo de afrontamiento distracción.

${ }^{*} \mathrm{p}<.05$

${ }^{* *} \mathrm{p}<.01$

${ }^{* * *} \mathrm{p}<.001$ 